\title{
A CLASS OF OPERATOR-VALUED MEROMORPHIC FUNCTIONS ON THE UNIT DISC
}

\author{
Peter Jonas \\ Technische Universität Berlin, Fachbereich Mathematik (MA 7-2) \\ Straße des 17. Juni Nr. 135, D-W-1000 Berlin 12, Germany
}

\begin{abstract}
Let $\mathscr{L}(\mathscr{H})$ denote the algebra of all bounded linear operators on a separable Hilbert space $\mathscr{H}$. The first objective of this note is to study the class $D_{0}(\mathscr{L}(\mathscr{H}))$ of all $\mathscr{L}(\mathscr{H})$-valued functions $F$ meromorphic in the open disc $\mathbf{D}$ and holomorphic at 0 , admitting a representation of the form

$$
F(z)=\left(\sum_{j=-N}^{N} g_{j} z^{j}\right)^{-1}\left\{\int_{0}^{2 \pi}\left(e^{i \theta}+z\right)\left(e^{i \theta}-z\right)^{-1} d \Sigma(\theta)+\sum_{j=-N}^{N} h_{j} z^{j}\right\}
$$

where $g_{j}=\overline{g_{-j}} \in \mathbf{C}, j=0, \ldots, N, \Sigma$ is a positive operator measure and $h_{j}=-\left(h_{-j}\right)^{*} \in \mathscr{L}(\mathscr{H})$, $j=0, \ldots, N$. Evidently, the class $D_{0}(\mathscr{L}(\mathscr{H}))$ contains the holomorphic operator-valued functions in $\mathbf{D}$ with non-negative real part. It also contains the Krein-Langer classes $C_{\kappa}, \kappa=0,1, \ldots$, of meromorphic operator functions [16].

If $J \in \mathscr{L}(\mathscr{H}), J=J^{-1}=J^{*}$, and $U$ is a definitizable unitary operator in the Krein space $(\mathscr{H},(J \cdot, \cdot) \mathscr{H})$, one can define the spectra of non-negative and non-positive type of $U$ and construct the spectral function of $U$ with the help of the function $z \mapsto J(U+z I)(U-z I)^{-1}$, which belongs to $D_{0}(\mathscr{L}(\mathscr{H}))$. For an arbitrary $F \in D_{0}(\mathscr{L}(\mathscr{H}))$ we define analogues of the spectra of non-negative and non-positive type of definitizable unitary operators in the same way. Multiplicity functions for these sets are defined with the help of an analogue of the spectral function.

The second objective of this paper is to study the behaviour of the "spectra of non-negative and non-positive type of $F$ " under perturbations of $F$. For $F$ in a fixed class $C_{\kappa}$ these sets depend continuously on $F$ with respect to the uniform weak convergence.
\end{abstract}

\section{Introduction}

Let $\mathscr{H}$ be a separable complex Hilbert space and let $\mathscr{L}(\mathscr{H})$ denote the algebra of all bounded linear operators on $\mathscr{H}$. For a Banach space $X$, let $\mathscr{R}_{0, \infty}(X)$ denote the set of all functions $z \mapsto \sum_{j \in \mathbf{Z}} c_{j} z^{j}$ of a complex variable $z$ with $c_{j} \in X$, where the sum is finite, $\mathscr{R}_{0, \infty}:=\mathscr{R}_{0, \infty}(\mathbf{C})$. The set of all $\mathscr{L}(\mathscr{H})$-valued functions meromorphic in the open unit disc $\mathbf{D}$ and holomorphic at 0 will be denoted by $M_{0}(\mathscr{L}(\mathscr{H}))$. Throughout this paper, all functions $F \in M_{0}(\mathscr{L}(\mathscr{H}))$ are tacitly assumed to be extrapolated to the exterior of the unit circle $\mathbf{T}$ such that $F\left(\bar{z}^{-1}\right)=-(F(z))^{*}, z \in \mathbf{D}$. The set of all points $z \in \mathbf{C}$ such that (the

1991 Mathematics Subject Classification: Primary 46F20; Secondary 30E05, 47A56.

doi:10.5186/aasfm.1992.1727 
extrapolated) $F$ cannot be analytically continued in $z$ is called the spectrum of $F$ and denoted by $\sigma(F)$.

Let $C_{0, \kappa}^{+}(\mathscr{L}(\mathscr{H}))$ be the class of all functions $F \in M_{0}(\mathscr{L}(\mathscr{H}))$ such that the kernel $C_{F}$,

$$
C_{F}(z, \zeta):=(1-z \bar{\zeta})^{-1}\left(F(z)+F(\zeta)^{*}\right),
$$

has exactly $\kappa, \kappa \in \mathbf{N}_{0}:=\mathbf{N} \cup\{0\}$, negative squares, i.e. for any positive integer $k$, any $z_{1}, \ldots, z_{k} \in \mathbf{D}$ in the domain of holomorphy of $F$ and $x_{1}, \ldots, x_{k} \in \mathscr{H}$ the matrix $\left(\left(C_{F}\left(z_{\nu}, z_{\mu}\right) x_{\nu}, x_{\mu}\right)\right)_{\nu, \mu=1, \ldots, k}$ has at most $\kappa$ negative eigenvalues and for at least one choice of $k, z_{1}, \ldots, z_{k}, x_{1}, \ldots, x_{k}$ it has exactly $\kappa$ negative eigenvalues. These classes of operator functions were introduced by M.G. Krein and H. Langer in [16]. For basic results on these classes see [16], [17], [18], [19], [4]. Recall that $C_{0,0}^{+}(\mathscr{L}(\mathscr{H}))$ coincides with the class of all holomorphic $\mathscr{L}(\mathscr{H})$-valued functions $F$ in $\mathbf{D}$ such that $\operatorname{Re} F(z) \geq 0, z \in \mathbf{D}$, or, equivalently, $F$ admits a representation

$$
F(z)=i S+(4 \pi)^{-1} \int_{0}^{2 \pi}\left(e^{i \theta}+z\right)\left(e^{i \theta}-z\right)^{-1} d \Sigma(\theta), \quad z \in \mathbf{D},
$$

where $S \in \mathscr{L}(\mathscr{H})$ is self-adjoint and $\Sigma$ is a positive operator measure in $\mathscr{H}$ (see e.g. [3]). If $F \in C_{0, \kappa}^{+}(\mathscr{L}(\mathscr{H}))$, then, as a consequence of a representation of $F$ by means of a unitary operator in a Pontryagin space $\Pi_{\kappa}$ (see [16]), $F$ can be written in the form

$$
F(z)=g(z)^{-1}\left\{\int_{0}^{2 \pi}\left(e^{i \theta}+z\right)\left(e^{i \theta}-z\right)^{-1} d \Sigma(\theta)+G(z)\right\},
$$

where $g \in \mathscr{R}_{0, \infty}, g(z) \geq 0$ if $|z|=1, G \in \mathscr{R}_{0, \infty}(\mathscr{L}(\mathscr{H}))$ and $\Sigma$ is a positive operator measure (see [19], Section 1.3). Simple examples show that not every function of the form (0.3) belongs to one of the classes $C_{0, \kappa}^{+}(\mathscr{L}(\mathscr{H})), \kappa=0,1, \ldots$

The first objective of this note is to study the class of all operator functions $F \in M_{0}\left(\mathscr{L}(\mathscr{H})\right.$ ) which admit a representation of the form $(0.3)$, where $g \in \mathscr{R}_{0, \infty}$ and $g$ is only assumed to be real on $\mathbf{T}, \Sigma$ is a positive operator measure and $G \in \mathscr{R}_{0, \infty}(\mathscr{L}(\mathscr{H}))$ (Section 2). We shall denote this class by $D_{0}(\mathscr{L}(\mathscr{H}))$. By definition, $D_{0}(\mathscr{L}(\mathscr{H}))$ contains the classes $C_{0, \kappa}^{+}(\mathscr{L}(\mathscr{H})), \kappa=0,1, \ldots$

In our study we shall largely make use of the fact that, by the well-known duality of spaces of locally holomorphic functions, for every function $F \in M_{0}(\mathscr{L}(\mathscr{H}))$ there is a corresponding continuous linear mapping $T_{F}$ of the space of locally holomorphic functions on $\sigma(F)$ in $\mathscr{L}(\mathscr{H})$ (see e.g. [14], [7]). This correspondence is one-to-one up to constant operator functions with a skew-self-adjoint value. If e.g. $F \in C_{0,0}^{+}(\mathscr{L}(\mathscr{H}))$ and $(0.2)$ holds, then $T_{F}$ is the Radon measure corresponding to $\Sigma$. 
For $F \in D_{0}(\mathscr{L}(\mathscr{H})), T_{F}$ restricted to $\mathbf{T}$ is an $\mathscr{L}(\mathscr{H})$-valued distribution, and the properties of $T_{F}$ are very similar to those of the functional calculus of a definitizable unitary operator $U$ in a Krein space. We remark that the functional calculus of such an operator $U$ is of the form $T_{F_{U}}$ for a certain operator function $F_{U} \in D_{0}(\mathscr{L}(\mathscr{H}))$ closely connected with the resolvent of $U$ (see Example 1.3). For $F \in D_{0}(\mathscr{L}(\mathscr{H}))$ we define, analogously to the operator case, i.e. by extension of $T_{F}$, a so-called spectral function of $F$.

Recall that, for a definitizable unitary operator $U$ in a Krein space, there exists a maximal non-negative (maximal non-positive) $U$-invariant subspace $\mathscr{M}_{+}$ (or $\left.\mathscr{M}_{-}\right)$such that $\sigma\left(U \mid \mathscr{M}_{+}\right) \subset \overline{\mathbf{D}}\left(\sigma\left(U \mid \mathscr{M}_{-}\right) \subset \overline{\mathbf{D}}\right)$, [20]. The sets $\sigma_{+}^{\mathbf{D}}(U):=$ $\sigma\left(U \mid \mathscr{M}_{+}\right)$and $\sigma_{-}^{\mathbf{D}}(U):=\sigma\left(U \mid \mathscr{M}_{-}\right)$which do not depend on the special choice of $\mathscr{M}_{+}$and $\mathscr{M}_{-}$can also be defined by making use of the functional calculus or the spectral function of $U$. We have $\sigma(U) \cap \overline{\mathbf{D}}=\sigma_{+}^{\mathbf{D}}(U) \cup \sigma_{-}^{\mathbf{D}}(U)$. This classification of spectral points is carried over to functions $F \in D_{0}(\overline{\mathscr{L}}(\mathscr{H}))$ by making use of $T_{F}$. The notion of multiplicity of an isolated point $\mu$ of $\sigma_{+}^{\mathbf{D}}(U)\left(\sigma_{-}^{\mathbf{D}}(U)\right)$ which is by definition the algebraic multiplicity of the eigenvalue $\mu$ of $U \mid \mathscr{M}_{+}\left(U \mid \mathscr{M}_{-}\right)$ can be carried over to the case of a function $F \in D_{0}(\mathscr{L}(\mathscr{H}))$ with the aid of a certain sesquilinear functional which is closely related to $T_{F}$.

The second objective of this note is to study the behaviour of $\sigma_{+}^{\mathbf{D}}(F)$ and $\sigma_{-}^{\mathbf{D}}(F)$ (the analogues of $\sigma_{+}^{\mathbf{D}}(U)$ and $\sigma_{-}^{\mathbf{D}}(U)$ ) under perturbations of $F$ (Section 3). For the case where $F$ belongs to a fixed class $C_{0, \kappa}^{+}(\mathscr{L}(\mathscr{H}))$ we prove that $\sigma_{-}^{\mathbf{D}}(F)$ depends continuously on $F$ with respect to uniform weak convergence in the neighbourhood of a point in $\mathbf{D}$. In this case $\sigma_{-}^{\mathbf{D}}(F)$ coincides with the union of the set of poles of $F$ in $\mathbf{D}$ and the set of the so-called generalized poles of negative type of $F$ ([19], Corollary 3.2).

With the exception of Proposition 3.5, we do not make use of representations of holomorphic operator functions by unitary operators in Krein spaces (see e.g. [5], [16]). Operator representations for functions of the class $D_{0}(\mathscr{L}(\mathscr{H}))$ will be considered in a subsequent paper.

By a linear fractional transformation of the independent variable all results of this note can be carried over to functions which are meromorphic in the open upper half-plane.

Remark for notation: $C^{m}(\mathbf{T}, \mathscr{H})$ denotes the space of $C^{m}$ functions on $\mathbf{T}$ with values in $\mathscr{H}$. Similar notation is used for other spaces of vector-valued functions.

I thank Professors H. Langer and I.S. Louhivaara for encouragement and critical help in the preparation of the manuscript. 


\section{Some functionals related to meromorphic operator functions on D}

1.1. Definition and basic properties. For any subset $\mathscr{S}$ of the extended complex plane $\overline{\mathbf{C}}$ we write $\hat{\mathscr{S}}:=\left\{z \in \overline{\mathbf{C}}: \bar{z}^{-1} \in s\right\}$. For any scalar (or $\mathscr{L}(\mathscr{H})$ valued) function $f$ defined on a set $\mathscr{D}=\hat{\mathscr{D}} \subset \overline{\mathbf{C}}$ we define $\hat{f}(\mu):=\overline{f\left(\bar{\mu}^{-1}\right)}$ $\left(\hat{f}(\mu):=f\left(\bar{\mu}^{-1}\right)^{*}\right), \mu \in \mathscr{D}$. By $\mathscr{R}_{0, \infty}^{s}\left(\mathscr{R}_{0, \infty}^{s}(\mathscr{L}(\mathscr{H}))\right)$ we denote the set of all functions $g \in \mathscr{R}_{0, \infty}\left(\mathscr{R}_{0, \infty}(\mathscr{L}(\mathscr{H}))\right.$, see Introduction) such that $g=\hat{g}$. We shall say that $g \in \mathscr{R}_{0, \infty}$ is of degree $\leq N$ if $g(z)=\sum_{j=-N}^{N} c_{j} z^{j}, c_{j} \in \mathbf{C}$. Let $H(K)$ denote the space of locally holomorphic functions on the compact set $K \subset \mathbf{C}$ equipped with the usual topology (see [15], Section 27.4).

Let $\sigma_{0}=\hat{\sigma}_{0}$ be a countable subset of $\mathbf{C} \backslash \mathbf{T}$ whose accumulation points belong to $\mathbf{T}$. We denote by $\Phi\left(\mathbf{T} \cup \sigma_{0}, \mathscr{L}(\mathscr{H})\right)$ the linear space of all

$$
T \in \mathscr{L}\left(H\left(\mathbf{T} \cup \sigma_{0}\right), \mathscr{L}(\mathscr{H})\right)
$$

(for these mappings we write $f \mapsto T$.f) such that the following holds:

(i) For every point $z_{0} \in \sigma_{0}$, the restriction of $T$ to the subspace of all functions $f \in H\left(\mathbf{T} \cup \sigma_{0}\right)$ which are zero in some neighbourhood of $\left(\mathbf{T} \cup \sigma_{0}\right) \backslash\left\{z_{0}\right\}$ has the form $f \mapsto \sum_{\nu=0}^{k} A_{\nu} f^{(\nu)}\left(z_{0}\right)$ where $A_{\nu} \in \mathscr{L}(\mathscr{H}), \nu=0, \ldots, k$.

(ii) $T . \hat{f}=(T . f)^{*}, f \in H\left(\mathbf{T} \cup \sigma_{0}\right)$.

We define a product of $T \in \Phi\left(\mathbf{T} \cup \sigma_{0}, \mathscr{L}(\mathscr{H})\right)$ and $g \in H\left(\mathbf{T} \cup \sigma_{0}\right), g=\hat{g}$ by $g T . f:=T . g f, f \in H\left(\mathbf{T} \cup \sigma_{0}\right)$.

With every function $F \in M_{0}(\mathscr{L}(\mathscr{H}))$ we connect certain analytic functionals. Set $\sigma_{0}(F):=\sigma(F) \backslash \mathbf{T}$. First we define an element $T_{F}$ of $\Phi\left(\mathbf{T} \cup \sigma_{0}(F), \mathscr{L}(\mathscr{H})\right)$ by

$$
T_{F} \cdot f:=-\int_{\mathscr{C}} F(z) f(z)(i z)^{-1} d z, \quad f \in H\left(\mathbf{T} \cup \sigma_{0}(F)\right),
$$

where $\mathscr{C}$ is the oriented boundary of a finite union $G$ of smooth domains containing $\mathbf{T} \cup \sigma_{0}(F)$ such that $f$ is defined on $\bar{G}$ and $0 \notin \bar{G}$. We have $T_{F_{1}}=T_{F_{2}}$ if and only if $F_{1}-F_{2} \equiv i S$ for some $S=S^{*} \in \mathscr{L}(\mathscr{H})$.

Every $T \in \Phi\left(\mathbf{T} \cup \sigma_{0}, \mathscr{L}(\mathscr{H})\right)$, where $\sigma_{0}$ is as above, is of the form $T_{F}$ for some $F \in M_{0}(\mathscr{L}(\mathscr{H}))$. Indeed, let $f_{\lambda}(z):=(2 \pi)^{-1} z(z-\lambda)^{-1}, \lambda \in \overline{\mathbf{C}} \backslash\left(\mathbf{T} \cup \sigma_{0}\right)$, and

$$
\tilde{T}(\lambda):=T \cdot f_{\lambda} .
$$

Then $\lambda \mapsto F(\lambda):=\tilde{T}(\lambda)-\frac{1}{2} \tilde{T}(0)$ belongs to $M_{0}(\mathscr{L}(\mathscr{H}))$ and we have $\sigma_{0} \supset \sigma_{0}(F)$ and $T=T_{F}$.

If $F \in M_{0}(\mathscr{L}(\mathscr{H}))$, then

$$
\tilde{T}_{F}(\lambda)=F(\lambda)-F(\infty), \quad \lambda \notin \mathbf{T} \cup \sigma_{0}(F),
$$


and

$$
\tilde{T}_{F}(\lambda)-\frac{1}{2} \tilde{T}_{F}(0)=F(\lambda)-i \operatorname{Im} F(0), \quad \lambda \notin \mathbf{T} \cup \sigma_{0}(F) .
$$

The correspondence between $F$ and $T_{F}$ is a special case of a more general correspondence considered in [7], Théorème 3 .

Furthermore, for every function $F \in M_{0}(\mathscr{L}(\mathscr{H}))$ and every pair $u, v \in$ $H\left(\mathbf{T} \cup \sigma_{0}(F), \mathscr{H}\right)$, we define an analytic functional $T_{F}(u, v) \in H\left(\mathbf{T} \cup \sigma_{0}(F)\right)^{\prime}$ by

$$
T_{F}(u, v) . f=-\int_{\mathscr{C}}\left(F(z) u(z), v\left(\bar{z}^{-1}\right)\right) f(z)(i z)^{-1} d z, f \in H\left(\mathbf{T} \cup \sigma_{0}(F)\right),
$$

where $\mathscr{C}$ is defined as in (1.1). For a related (more general) functional see [7], Théorème 2. The sesquilinear form $(u, v) \mapsto T_{F}(u, v) . f$ in $H\left(\mathbf{T} \cup \sigma_{0}(F), \mathscr{H}\right)$ is Hermitian if $f=\hat{f}$. Furthermore, we have

$$
\begin{gathered}
T_{F}(g u, v) . f=T_{F}(u, \hat{g} v) . f=T_{F}(u, v) . g f=: g T_{F}(u, v) . f, \\
f, g \in H\left(\mathbf{T} \cup \sigma_{0}(F)\right), \quad u, v \in H\left(\mathbf{T} \cup \sigma_{0}(F), \mathscr{H}\right) .
\end{gathered}
$$

It is often sufficient to consider $T_{F}(\cdot, \cdot)$ on $\mathscr{R}_{0, \infty}(\mathscr{H})$, which is a dense linear subspace of $H\left(\mathbf{T} \cup \sigma_{0}(F), \mathscr{H}\right)$. Let $u, v \in \mathscr{R}_{0, \infty}(\mathscr{H})$,

$$
u(z)=\sum_{\nu=-N}^{N} x_{\nu} z^{\nu}, \quad v(z)=\sum_{\mu=-N}^{N} y_{\mu} z^{\mu} .
$$

Then, for every $f \in H\left(\mathbf{T} \cup \sigma_{0}(F)\right)$, we have

$$
T_{F}(u, v) \cdot f=\sum_{\nu, \mu=-N}^{N}\left(\left(T_{F} \cdot p_{\nu-\mu} f\right) x_{\nu}, y_{\mu}\right),
$$

where $p_{k}(z):=z^{k}, k=0, \pm 1, \ldots$

The Hermitian form $T_{F}(\cdot, \cdot) .1$ is connected with the kernel $C_{F}$ (see (0.1)) by the relation

$$
2 \pi\left(C_{F}\left(z_{i}, z_{j}\right) x_{i}, x_{j}\right)=T_{F}\left(u_{i}, u_{j}\right) .1,
$$

where $z_{i}$ belong to the domain of holomorphy of $F, x_{i} \in \mathscr{H}, u_{i}(z):=\left(z-z_{i}\right)^{-1} x_{i}$, $i=1,2, \ldots, n$. We remark that the set of the functions of the form

$$
u(z)=\sum_{i=1}^{n}\left(z-z_{i}\right)^{-1} x_{i}
$$


is dense in $H\left(\mathbf{T} \cup \sigma_{0}(F), \mathscr{H}\right)$.

Assume now, in addition, that $F$ has no more than a finite number of poles in $\mathbf{D}$. Then, by $H_{0}\left(\mathbf{T} \cup \sigma_{0}(F)\right)\left(H_{1}\left(\mathbf{T} \cup \sigma_{0}(F)\right)\right)$, we denote the closed linear subspace of $H\left(\mathbf{T} \cup \sigma_{0}(F)\right)$ consisting of all $f \in H\left(\mathbf{T} \cup \sigma_{0}(F)\right)$ which are zero on a neighbourhood of $\mathbf{T}$ (or $\sigma_{0}(F)$ ). The natural isomorphisms of $H(\mathbf{T})$ onto $H_{1}\left(\mathbf{T} \cup \sigma_{0}(F)\right)$ and of $H(\mathbf{T}, \mathscr{H})$ onto $H_{1}\left(\mathbf{T} \cup \sigma_{0}(F), \mathscr{H}\right)$ are denoted by $\iota$. We define

$$
t_{F} . f:=T_{F} . \iota f, \quad f \in H(\mathbf{T}),
$$

and

$$
t_{F}(u, v) . f:=T_{F}(\iota u, \iota v) . \iota f, \quad u, v \in H(\mathbf{T}, \mathscr{H}), \quad f \in H(\mathbf{T}) .
$$

Then one verifies without difficulty that $T_{F}$ has the form

(1.7) $T_{F} \cdot f=t_{F} \cdot f+\sum_{i=1}^{k} \sum_{j=0}^{r_{i}-1}\left\{F_{i j} f^{[j]}\left(\mu_{i}\right)+F_{i j}^{*} f^{[j]}\left(\bar{\mu}_{i}^{-1}\right)\right\}, \quad f \in H\left(\mathbf{T} \cup \sigma_{0}(F)\right)$,

where

$$
f^{[0]}(z):=f(z), \quad f^{[j]}(z)=i z\left(d f^{[j-1]} / d z\right)(z), \quad j=1,2, \ldots,
$$

$\mu_{1}, \ldots, \mu_{k}$ are the poles of $F$ in $\mathbf{D}, r_{1}, \ldots, r_{k}$ their orders, respectively, and $F_{i j} \in \mathscr{L}(\mathscr{H})$.

We denote by $M_{0}^{m}(\mathscr{L}(\mathscr{H})), m=1,2, \ldots$, the set of all $F \in M_{0}(\mathscr{L}(\mathscr{H}))$ with finite $\sigma_{0}(F)$ such that

$$
\sup \left\{\|F(z)\||1-| z||^{m}:|z| \in(\eta, 1) \cup\left(1, \eta^{-1}\right)\right\}<\infty
$$

for some $\eta \in(0,1)$. We set $M_{0}^{\infty}(\mathscr{L}(\mathscr{H})):=\bigcup_{m=1}^{\infty} M_{0}^{m}(\mathscr{L}(\mathscr{H}))$.

The following proposition is well known [14].

Proposition 1.1. Let $F \in M_{0}(\mathscr{L}(\mathscr{H}))$ and let $\sigma_{0}(F)$ be finite. Then we have $F \in M_{0}^{\infty}(\mathscr{L}(\mathscr{H}))$ if and only if $t_{F}$ is a $\mathscr{L}(\mathscr{H})$-valued distribution on $\mathbf{T}$.

Let $F \in M_{0}^{m}(\mathscr{L}(\mathscr{H}))$ for some positive integer $m$. Then $t_{F}$ can be extended by continuity to an element of $\mathscr{L}\left(C^{m+1}(\mathbf{T}), \mathscr{L}(\mathscr{H})\right)$. For every $f \in C^{m+1}(\mathbf{T})$ and every $q \in \mathscr{R}_{0, \infty}^{s}$ we have

$$
\begin{aligned}
q t_{F} . f & =\lim _{r \uparrow 1} \int_{0}^{2 \pi}\left(q\left(r e^{i \theta}\right) F\left(r e^{i \theta}\right)-q\left(r^{-1} e^{i \theta}\right) F\left(r^{-1} e^{i \theta}\right)\right) f\left(e^{i \theta}\right) d \theta \\
& =\lim _{r \uparrow 1} 2 \int_{0}^{2 \pi} \operatorname{Re}\left\{q\left(r e^{i \theta}\right) F\left(r e^{i \theta}\right)\right\} f\left(e^{i \theta}\right) d \theta
\end{aligned}
$$

where the limits and integrals are understood in the sense of the weak operator topology. 
It follows from (1.10) that for $F \in M_{0}^{m}(\mathscr{L}(\mathscr{H}))$ and a real function $f \in$ $C^{m+1}(\mathbf{T})$ the operator $t_{F} \cdot f$ is self-adjoint.

Now we shall prove similar properties for the functional $t_{F}(\cdot, \cdot)$.

Proposition 1.2. Let $F \in M_{0}^{m}(\mathscr{L}(\mathscr{H}))$ for some positive integer $m$. Then:

(i) The mapping $(u, v, f) \mapsto t_{F}(u, v) . f$ is continuous with respect to the topology of $C^{m+1}(\mathbf{T}, \mathscr{H}) \times C^{m+1}(\mathbf{T}, \mathscr{H}) \times C^{m+1}(\mathbf{T})$. Hence this mapping can be extended to this space by continuity.

(ii) For every $q \in \mathscr{R}_{0, \infty}^{s}, u, v \in C^{m+1}(\mathbf{T}, \mathscr{H}), f \in C^{m+1}(\mathbf{T})$ we have

$$
\begin{aligned}
q t_{F}(u, v) . f & =\lim _{r \uparrow 1} \int_{0}^{2 \pi}\left(\left(q\left(r e^{i \theta}\right) F\left(r e^{i \theta}\right)-q\left(\frac{1}{r} e^{i \theta}\right) F\left(\frac{1}{r} e^{i \theta}\right)\right) u\left(e^{i \theta}\right), v\left(e^{i \theta}\right)\right) f\left(e^{i \theta}\right) d \theta \\
& =\lim _{r \uparrow 1} 2 \int_{0}^{2 \pi}\left(\operatorname{Re}\left\{q\left(r e^{i \theta}\right) F\left(r e^{i \theta}\right)\right\} u\left(e^{i \theta}\right), v\left(e^{i \theta}\right)\right) f\left(e^{i \theta}\right) d \theta .
\end{aligned}
$$

Proof. Assume that $u, v \in H(\mathbf{T}, \mathscr{H})$ and $f \in H(\mathbf{T})$ are defined for $|z| \epsilon$ $\left(\eta, \eta^{-1}\right)$, where $\eta$ is as in (1.9). Then we have

$$
t_{F}(u, v) \cdot f=-\int_{\mathscr{C}}\left(F(z) u(z), v\left(\bar{z}^{-1}\right)\right) f(z)(i z)^{-1} d z
$$

where, for $\mathscr{C}$ we may take e.g. the boundary of $\left\{z: \frac{1}{2}(\eta+1) \leq|z| \leq 2(\eta+1)^{-1}\right\}$. On account of (1.9) there exists an $A \in \mathscr{L}(\mathscr{H})$ and a holomorphic $\mathscr{L}(\mathscr{H})$-valued function $G_{i}$ on $\{z: \eta<|z|<1\}$ such that

(i) $G_{i}^{[m+1]}(z)=F(z)+A($ see $(1.8))$,

(ii) the function $G_{i}$ can be extended by continuity to a continuous function on $\{z: \eta \leq|z| \leq 1\}$ (see e.g. [14]; proof of Satz 19).

In $\left\{z: 1<|z|<\eta^{-1}\right\}$ there exists a function $G_{e}$ with similar properties. We set $G(z):=G_{i}(z)$ if $|z| \in(\eta, 1)$ and $G(z):=G_{e}(z)$ if $|z| \in\left(1, \eta^{-1}\right)$. Now we easily find that

$$
\begin{aligned}
(1.12)\left\{\left(G^{[m]}\right.\right. & \left.\left.(z) u(z), v\left(\bar{z}^{-1}\right)\right) f(z)\right\}^{[1]}(i z)^{-1} \\
= & \left(G^{[m+1]}(z) u(z), v\left(\bar{z}^{-1}\right)\right) f(z)(i z)^{-1} \\
& +\left(G^{[m]}(z) u^{[1]}(z), v\left(\bar{z}^{-1}\right)\right) f(z)(i z)^{-1} \\
& +\left(G^{[m]}(z) u(z), v^{[1]}\left(\bar{z}^{-1}\right)\right) f(z)(i z)^{-1} \\
& +\left(G^{[m]}(z) u(z), v\left(\bar{z}^{-1}\right)\right) f^{[1]}(z)(i z)^{-1}, \quad|z| \in(\eta, 1) \cup\left(1, \eta^{-1}\right) .
\end{aligned}
$$


The integral along $\mathscr{C}$ of the left-hand side of (1.12) is zero. By (1.12) and similar relations the right-hand side of (1.11) is equal to

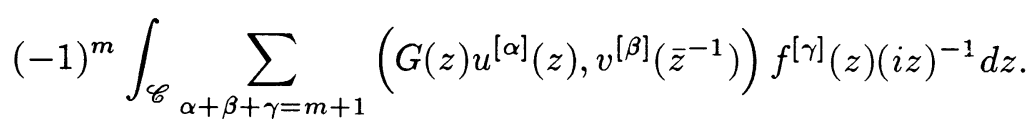

Making use of the continuity of $G_{i}$ and $G_{e}$ up to the unit circle, this integral can be expressed as an integral along the unit circle. This implies the continuity statement of (i). We can prove (ii) in a similar way.

It follows from Proposition 1.2 that, for fixed $u, v \in C^{m+1}(\mathbf{T}, \mathscr{H}), t_{F}(u, v)$ is a distribution on $\mathbf{T}$. For fixed $f \in C^{m+1}(\mathbf{T}),(u, v) \mapsto t_{F}(u, v) . f$ is a sesquilinear form on $C^{m+1}(\mathbf{T}, \mathscr{H})$ and we have

$$
t_{F}(u, v) . g f=t_{F}(g u, v) . f=t_{F}(u, \bar{g} v) . f, \quad f, g \in C^{m+1}(\mathbf{T}) .
$$

The form $(u, v) \mapsto t_{F}(u, v) . f$ is Hermitian if $f$ is real.

Example 1.3. Assume that on the Hilbert space $\mathscr{H}$ there is given an Hermitian sesquilinear form $[\cdot, \cdot]$ such that $|[x, y]| \leq c\|x\|\|y\|, x, y \in \mathscr{H}$, for some constant $c$. Let $W$ be the Gram operator of $[\cdot, \cdot]$,

$$
(W x, y)=[x, y], \quad x, y \in \mathscr{H},
$$

and let $U$ be a bounded and boundedly invertible operator such that every point of $\sigma(U) \backslash \mathbf{T}$ is a pole of the resolvent of $U$ and $[U x, U y]=[x, y], x, y \in \mathscr{H}$. Then the function $F_{U}$ defined by

$$
F_{U}(z):=W(U+z I)(U-z I)^{-1}=-W+2 W U(U-z I)^{-1}, \quad z \in \varrho(U),
$$

belongs to $M_{0}(\mathscr{L}(\mathscr{H}))$ and satisfies the relation $F_{U}(z)=-\left(F_{U}\left(\bar{z}^{-1}\right)\right)^{*}, z \in$ $\varrho(U)$.

The functional $T_{F_{U}}$ is closely connected with the Riesz-Dunford functional calculus of $U$; we have

$$
T_{F_{U}} \cdot f=4 \pi W f(U), \quad f \in H(\mathbf{T} \cup \sigma(U)) .
$$

If, in addition, $\sigma(U) \backslash \mathbf{T}$ is a finite set and $(U-z I)^{-1}$ satisfies a growing condition similar to (1.5), the Riesz-Dunford functional calculus can be extended by continuity to $C^{\infty}(\mathbf{T}) \times H(\sigma(U) \backslash \mathbf{T})$, we have $F_{U} \in M_{0}^{\infty}(\mathscr{L}(\mathscr{H}))$ and (1.15) holds for every $f \in C^{\infty}(\mathbf{T}) \times H(\sigma(U) \backslash \mathbf{T})$. If $0 \in \varrho(W)$ holds, or, equivalently, $(\mathscr{H},[\cdot, \cdot])$ is a Krein space, we shall replace below the letter $W$ with $G$. In this case we have $\sigma(U)=\sigma\left(F_{U}\right)$. 
1.2. Ranks of positivity and negativity of $T_{F}(\cdot, \cdot) . f$. If $\mathscr{L}$ is a linear space equipped with a Hermitian sesquilinear form $[\cdot, \cdot]$, we denote by $\kappa_{+}((\mathscr{L},[\cdot, \cdot]))\left(\kappa_{-}((\mathscr{L},[\cdot, \cdot]))\right)$ the least upper bound $(\leq \infty)$ of the dimensions of $[\cdot, \cdot]$-positive definite (or $[\cdot, \cdot]$-negative definite) subspaces of $\mathscr{L}$. These quantities are called the ranks of positivity and negativity of $[\cdot, \cdot]$ on $\mathscr{L}$.

If $F \in M_{0}(\mathscr{L}(\mathscr{H}))$ and $f \in H\left(\mathbf{T} \cup \sigma_{0}(F)\right)$ with $f=\hat{f}$, we define

$$
\kappa_{ \pm}(f ; F):=\kappa_{ \pm}\left(\left(\mathscr{R}_{0, \infty}(\mathscr{H}), T_{F}(\cdot, \cdot) \cdot f\right)\right) \text {. }
$$

In this definition $\mathscr{R}_{0, \infty}(\mathscr{H})$ can be replaced with $H\left(\mathbf{T} \cup \sigma_{0}(F), \mathscr{H}\right)$.

Let $F \in M_{0}^{m}(\mathscr{L}(\mathscr{H}))$ for some positive integer $m$. Then we define $\kappa_{ \pm}(f ; F)$ for every $f \in C^{m+1}(\mathbf{T}) \times H\left(\sigma_{0}(F)\right)$ with $f=\hat{f}$ by the same relation. In this case $\mathscr{R}_{0, \infty}(\mathscr{H})$ can be replaced, in view of Proposition 1.2 , (i), with $C^{m+1}(\mathbf{T} ; \mathscr{H}) \times$ $H\left(\sigma_{0}(F), \mathscr{H}\right)$ or with dense subspaces of this space.

By (1.6) a function $F \in M_{0}(\mathscr{L}(\mathscr{H}))$ belongs to $C_{0, \kappa}^{+}(\mathscr{L}(\mathscr{H}))$ (see Introduction) if and only if $\kappa_{-}(\mathbf{1} ; F)=\kappa$.

Let $F \in M_{0}^{\infty}(\mathscr{L}(\mathscr{H}))$. We shall say that an open subset $\gamma$ of $\mathbf{T}$ is of positive type (negative type, type $\pi_{+}$, type $\pi_{-}$) with respect to $F$ if $\kappa_{-}(f ; F)=0$ $\left(\kappa_{+}(f ; F)=0, \kappa_{-}(f ; F)<\infty, \kappa_{+}(f ; F)<\infty\right)$ for all non-negative functions $f \in C^{\infty}(\mathbf{T}) \times H\left(\sigma_{0}(F)\right)$ with supp $f \subset \gamma$.

Example 1.4. Let $[\cdot, \cdot], U$ and $F_{U}$ be as in Example 1.3 and let $F_{U} \in$ $M_{0}^{\infty}(\mathscr{L}(\mathscr{H}))$. Then it is easy to see that for every $f \in C^{\infty}(\mathbf{T}) \times H(\sigma(U) \backslash \mathbf{T})$ we have

$$
\kappa_{ \pm}\left(f ; F_{U}\right)=\kappa_{ \pm}((\mathscr{H},[f(U) \cdot, \cdot])) .
$$

In particular, if $(\mathscr{H},[\cdot, \cdot])$ is a Krein space, an open $\operatorname{arc} \gamma$ of $\mathbf{T}$ is of positive (negative) type with respect to $F_{U}$ if and only if it is of positive (negative) type with respect to $U$ (see [11], Section 2.1 and especially Proposition 2.1).

Lemma 1.5. Let $F \in M_{0}(\mathscr{L}(\mathscr{H})), \nu \in \sigma_{0}(F) \cap \mathbf{D}$ and let $l$ be the multiplicity of the pole $\nu$ of $F$. Assume that $\chi \in H\left(\mathbf{T} \cup \sigma_{0}(F)\right)$ is equal to 1 in an open neighbourhood $\mathfrak{U}$ of $\left\{\nu, \bar{\nu}^{-1}\right\}$ and equal to 0 in an open neighbourhood of $\left(\mathbf{T} \cup \sigma_{0}(F)\right) \backslash\left\{\nu, \bar{\nu}^{-1}\right\}$. Then $\kappa_{+}(\chi ; F)=\kappa_{-}(\chi ; F)=l$.

Proof. Let

$$
F(z)=(z-\nu)^{-k} A_{-k}+\cdots+(z-\nu)^{-1} A_{-1}+A_{0}+\cdots
$$

be the Laurent expansion of $F$ at $\nu$. Then $l$ is equal to the dimension of the range of the operator

$$
\left(\begin{array}{ccccc}
A_{-k} & 0 & \ldots & 0 & 0 \\
A_{-k+1} & A_{-k} & \ldots & 0 & 0 \\
\vdots & \vdots & \ddots & \vdots & \vdots \\
A_{-2} & A_{-3} & \ldots & A_{-k} & 0 \\
A_{-1} & A_{-2} & \ldots & A_{-k+1} & A_{-k}
\end{array}\right)
$$


in $\mathscr{H}^{k}$ (see [17], Lemma 4.1).

The linear subspace of $H\left(\mathbf{T} \cup \sigma_{0}(F)\right)$ of all functions $u \in H\left(\mathbf{T} \cup \sigma_{0}(F)\right)$ which are zero outside $\mathfrak{U} \cap \mathbf{D}(\mathfrak{U} \cap \widehat{\mathbf{D}})$ is denoted by $H^{i}\left(H^{e}\right)$. Now developing arbitrary functions $u_{i} \in H^{i}$ and $u_{e} \in H^{e}$ in Taylor series at $\nu$ and $\bar{\nu}^{-1}$, respectively, and putting them and the relation (1.17) into (1.4) we find this to hold: If $l$ is finite, there exist linearly independent systems of functions $u_{i, \alpha} \in H^{i}, \alpha=1, \ldots, l$, and $u_{e, \beta} \in H^{e}, \beta=1, \ldots, l$, such that

$$
T_{F}\left(u_{i, \alpha}, u_{e, \beta}\right) \cdot \chi=\delta_{\alpha, \beta}, \quad \alpha, \beta=1, \ldots, l,
$$

and there are no such systems consisting of more than $l$ functions. Therefore, in view of the relation

$$
\begin{aligned}
T_{F}\left(u_{i}+u_{e}, u_{i}+u_{e}\right) \cdot \chi & =T_{F}\left(u_{i}, u_{e}\right) \cdot \chi+T_{F}\left(u_{e}, u_{i}\right) \cdot \chi \\
& =2 \operatorname{Re} T_{F}\left(u_{i}, u_{e}\right) \cdot \chi, \quad u_{i} \in H^{i}, u_{e} \in H^{e}
\end{aligned}
$$

the ranks of positivity and negativity of the form $(u, v) \mapsto T_{F}(u, v) \cdot \chi$ are equal to $l$. If $l=\infty$, then, for every positive integer $l^{\prime}$, there exist systems of functions $u_{i, \alpha} \in H^{i}, u_{e, \beta} \in H^{e}, \alpha, \beta=1, \ldots, l^{\prime}$, which satisfy a relation analogous to (1.18). Hence $\kappa_{+}(\chi ; F)=\kappa_{-}(\chi ; F)=\infty$.

Remark 1.6. Lemma 1.5 implies the well-known fact that, for a function $F \in C_{0, \kappa}^{+}(\mathscr{L}(\mathscr{H}))$, the total multiplicity of the poles of $F$ in $\mathbf{D}$ is not greater than $\kappa$.

1.3. Decompositions of $F$. Moments of $T_{F}$. Let $F \in M_{0}^{\infty}(\mathscr{L}(\mathscr{H}))$. Assume that $\gamma_{j}, j=1, \ldots, l$, are open arcs of $\mathbf{T}$ with $\bigcup_{j} \gamma_{j}=\mathbf{T}$, and let $\chi_{j} \in C^{\infty}(\mathbf{T}) \times H\left(\sigma_{0}(F)\right), j=1, \ldots, l$, such that $0 \leq \chi_{j}(s) \leq 1(s \in \mathbf{T})$, $\operatorname{supp} \chi_{j} \subset \gamma_{j}$ and $\sum_{j=1}^{l} \chi_{j}=1$ on $\mathbf{T}$. Let $\chi_{0} \in H_{0}\left(\mathbf{T} \cup \sigma_{0}(F)\right)$ be equal to 1 on a neighbourhood of $\sigma_{0}(F)$. Then, according to (1.3), we have

$$
F(\lambda)=i \operatorname{Im} F(0)+\sum_{j=0}^{l}\left(\chi_{j} T_{F} \cdot f_{\lambda}-(4 \pi)^{-1} \chi_{j} T_{F} \cdot \mathbf{1}\right) .
$$

The functions $\lambda \mapsto \chi_{j} T_{F} \cdot f_{\lambda}-(4 \pi)^{-1} \chi_{j} T_{F} .1=: F_{j}(\lambda), j=0, \ldots, l$, belong to $M_{0}^{\infty}(\mathscr{L}(\mathscr{H}))$, and we have

$$
T_{F_{j}}=\chi_{j} T_{F} \quad \text { and } \quad T_{F_{j}}(\cdot, \cdot)=\chi_{j} T_{F}(\cdot, \cdot) .
$$

Moreover, we have

$$
\sigma\left(F_{0}\right)=\sigma_{0}(F), \quad \sigma\left(F_{j}\right) \subset \sigma(F) \cap \operatorname{supp} \chi_{j}, \quad j=1, \ldots, l .
$$


We shall need some relations for the moments $\varphi_{l}:=(2 \pi)^{-1} T_{F} \cdot p_{-l}, p_{l}(z):=$ $z^{l}, l=0, \pm 1, \ldots$, of $T_{F}$. Here we only assume that $F \in M_{0}(\mathscr{L}(\mathscr{H}))$. The relations (1.2) and (1.3) imply

$$
\left\{\begin{array}{l}
\varphi_{0}=(2 \pi)^{-1} T_{F} \cdot 1=2 \operatorname{Re} F(0), \\
\varphi_{l}=(2 \pi)^{-1} T_{F} \cdot p_{-l}= \begin{cases}\left.l !^{-1} T_{F} \cdot \frac{d^{l}}{d \lambda^{l}} f_{\lambda}\right|_{\lambda=0}=l !^{-1} F^{(l)}(0) & \text { if } l \geq 1, \\
|l| !^{-1}\left(F^{(|l|)}(0)\right)^{*} & \text { if } l \leq-1 .\end{cases}
\end{array}\right.
$$

If $u(z):=\sum_{j=-n}^{n} x_{j} z^{j}, x_{j} \in \mathscr{H}$, we have by $(1.21)$

$$
(2 \pi)^{-1} T_{F}(u, u) \cdot \mathbf{1}=\sum_{j, k=-n}^{n}\left(\varphi_{k-j} x_{j}, x_{k}\right) .
$$

If $g \in \mathscr{R}_{0, \infty}^{s}, g(z)=\sum_{k=-N}^{N} g_{k} z^{k}$, then, by (1.21),

$$
(2 \pi)^{-1} g T_{F} \cdot p_{-l}=\sum_{k=-N}^{N} g_{k} \varphi_{l-k} .
$$

1.4. Characterization of arcs of positive and negative type. In the following lemma we characterize the open arcs of positive type in different ways. A similar result holds for open arcs of negative type.

Lemma 1.7. Let $F \in M_{0}^{\infty}(\mathscr{L}(\mathscr{H}))$ and let $\gamma$ be either an open arc of $\mathbf{T}$ or $\gamma=\mathbf{T}$. Then the following conditions are equivalent:

(i) $\gamma$ is of positive type with respect to $F$.

(ii) $t_{F}$ restricted to $\gamma$ is a positive measure, i.e. for every non-negative $f \in$ $C^{\infty}(\mathbf{T})$ with supp $f \subset \gamma$ the operator $t_{F} . f$ is non-negative.

(iii) There exists a $g_{0} \in C^{\infty}(\mathbf{T}), g_{0}\left(e^{i \theta}\right)>0$ if $e^{i \theta} \in \gamma$ such that the form $(u, v) \mapsto t_{F}(u, v) . g_{0}$ is non-negative semi-definite.

(iv) The following two conditions are satisfied for every $x \in \mathscr{H}$ :

( $\alpha) \liminf _{r \uparrow 1}\left(\left(F\left(r e^{i \theta}\right)-F\left(r^{-1} e^{i \theta}\right)\right) x, x\right) \geq 0$ for almost every $e^{i \theta} \in \gamma$.

$(\beta) \inf \left\{\left(\left(F\left(r e^{i \theta}\right)-F\left(r^{-1} e^{i \theta}\right)\right) x, x\right): e^{i \theta} \in \gamma_{0}, r \in(1-\delta, 1)\right\}>-\infty$ for every closed subarc $\gamma_{0}$ of $\gamma$ and sufficiently small $\delta>0$.

Proof. We prove the lemma for $\gamma \neq \mathbf{T}$. A similar reasoning applies if $\gamma=\mathbf{T}$. Evidently, (i) implies (ii).

(ii) $\Rightarrow$ (i): Assume that (ii) holds. Let $g$ be an arbitrary non-negative function belonging to $C^{\infty}(\mathbf{T}) \times H\left(\sigma_{0}(F)\right)$ with supp $g \subset \gamma$. Set $\gamma_{1}:=\gamma$ and let $\gamma_{2}$ be an open arc of $\mathbf{T}$ such that $\gamma_{1} \cup \gamma_{2}=\mathbf{T}$ and $\gamma_{2} \cap \operatorname{supp} g=\emptyset$. Then we decompose $F$ as in (1.19):

$$
F(\lambda)=i \operatorname{Im} F(0)+F_{0}(\lambda)+F_{1}(\lambda)+F_{2}(\lambda) .
$$


Then, by the relations (1.20), $T_{F_{1}}$ is a positive measure and $T_{F}(\cdot, \cdot) \cdot g=T_{F_{1}}(\cdot, \cdot) \cdot g$. If $u \in \mathscr{R}_{0, \infty}(\mathscr{H})$, Proposition 1.2 gives

$$
T_{F_{1}}(u, u) \cdot g=\lim _{r \uparrow 1} \int_{0}^{2 \pi}\left(\left\{\tilde{T}_{F_{1}}\left(r e^{i \theta}\right)-\tilde{T}_{F_{1}}\left(\frac{1}{r} e^{i \theta}\right)\right\} u\left(e^{i \theta}\right), u\left(e^{i \theta}\right)\right) g\left(e^{i \theta}\right) d \theta .
$$

Since the operators

$$
\tilde{T}_{F_{1}}\left(r e^{i \theta}\right)-\tilde{T}_{F_{1}}\left(\frac{1}{r} e^{i \theta}\right),
$$

which are Abel-Poisson integrals of the positive measure $T_{F_{1}}$, are non-negative, we conclude that $T_{F_{1}}(u, u) . g \geq 0$. Hence the assertion (i) holds.

That (ii) is equivalent to (iv) follows by a reasoning similar to the proof of (ii) $\Rightarrow$ (i) and by making use of the Lebesgue Theorem.

(ii) $\Rightarrow$ (iii): Assume that (ii) holds. Let $g_{0} \in C^{\infty}(\mathbf{T})$ such that $g_{0} \mid \mathbf{T} \backslash \gamma \equiv 0$ and $g_{0}\left(e^{i \theta}\right)>0$ if $e^{i \theta} \in \gamma$. Then $g_{0} t_{F}$ is a positive measure on $\mathbf{T}$. Set $F_{1}(\lambda):=$ $g_{0} t_{F} \cdot f_{\lambda}-(4 \pi)^{-1} g_{0} t_{F} \cdot \mathbf{1}$. Then we see as above that $t_{F}(u, u) \cdot g_{0}=T_{F_{1}}(u, u) . \mathbf{1}$ is non-negative for all $u \in \mathscr{R}_{0, \infty}(\mathscr{H})$.

(iii) $\Rightarrow$ (ii): Assume that (iii) holds. For every $p \in \mathscr{R}_{0, \infty}$ we have

$$
\left(\left(t_{F} \cdot p \hat{p} g_{0}\right) x, x\right)=t_{F}(x, x) \cdot p \hat{p} g_{0}=t_{F}(p x, p x) \cdot g_{0} \geq 0, \quad x \in \mathscr{H} .
$$

Then (ii) follows from the fact that, for an arbitrary positive integer $m$, every non-negative $C^{m}$ function on $\mathbf{T}$ is the limit in $C^{m}(\mathbf{T})$ of a sequence of functions of the form $p \hat{p}$.

\section{The class $D_{0}(\mathscr{L}(\mathscr{H}))$}

2.1. Characterizations of the class $D_{0}(\mathscr{L}(\mathscr{H}))$. In what follows we are concerned with the subclass $D_{0}(\mathscr{L}(\mathscr{H}))$ of $M_{0}(\mathscr{L}(\mathscr{H}))$ defined above in the introduction. The subclass $D_{0}(\mathscr{L}(\mathscr{H}))$ is the set of all $F \in M_{0}(\mathscr{L}(\mathscr{H}))$ satisfying a relation of the form

$$
g(z) F(z)=H(z)+i h(z), \quad z \in \mathbf{D},
$$

where $g \in \mathscr{R}_{0, \infty}^{s}, H$ is holomorphic in $\mathbf{D}, \operatorname{Re} H(z)=\frac{1}{2}\left(H(z)+H(z)^{*}\right) \geq 0$, $z \in \mathbf{D}$, and $h \in \mathscr{R}_{0, \infty}^{s}(\mathscr{L}(\mathscr{H}))$. A function $g \in \mathscr{R}_{0, \infty}^{s}$ with this property is called a definitizing function for $F$. By means of the Laurent expansions at 0 of the functions occurring in (2.1) we see that $H$ and $h$ are uniquely determined by $F$ and $g$ if we require, in addition, that $\operatorname{Im} H(0)=0$. If, in this case, $g(z)=$ $\sum_{j=-N}^{N} g_{j} z^{j}$, then $h(z)=\sum_{j=-N}^{N} h_{j} z^{j}$, where

$$
\left\{\begin{array}{l}
h_{0}=\operatorname{Im}\left\{\sum_{\nu=0}^{N}(\nu !)^{-1} g_{-\nu} F^{(\nu)}(0)\right\}, \\
h_{j}=h_{-j}^{*}=i \sum_{\nu=j}^{N}((\nu-j) !)^{-1} \overline{g_{-\nu}}\left(F^{(\nu-j)}(0)\right)^{*}, \quad 0<j \leq N .
\end{array}\right.
$$


Remark 2.1. Let $F \in M_{0}(\mathscr{L}(\mathscr{H}))$. If there exists a $g \in \mathscr{R}_{0, \infty}^{s}$ such that

$$
g(z) F(z)=E(z)+i e(z)
$$

where $E \in D_{0}(\mathscr{L}(\mathscr{H}))$ and $e \in \mathscr{R}_{0, \infty}^{s}(\mathscr{L}(\mathscr{H}))$, then $F \in D_{0}(\mathscr{L}(\mathscr{H}))$. This immediately follows from the definition of the class $D_{0}(\mathscr{L}(\mathscr{H}))$.

Proposition 2.2. For any $F \in M_{0}(\mathscr{L}(\mathscr{H}))$ the following assertions are equivalent:

(i) $F \in D_{0}(\mathscr{L}(\mathscr{H}))$.

(ii) There exists a $g \in \mathscr{R}_{0, \infty}^{s}$ such that $\operatorname{supp}\left(g T_{F}\right) \subset \mathbf{T}$ and $g t_{F}$ is a positive measure on $\mathbf{T}$.

(iii) $F \in M_{0}^{\infty}(\mathscr{L}(\mathscr{H}))$. There exists a finite set $e \subset \mathbf{T}$ such that all subarcs of $\mathbf{T} \backslash e$ are either of positive or of negative type with respect to $F$.

(iv) There exist $g_{l} \in \mathbf{C}, g_{l}=\overline{g_{-l}}, l=-N, \ldots, N$, such that the operator Toeplitz matrix $\left(\sum_{l=-N}^{N} g_{l} \varphi_{k-j-l}\right)_{k, j=-n}^{n}$, where $\varphi_{i}=(2 \pi)^{-1} T_{F} \cdot p_{-i}, \quad i=$ $0, \pm 1, \ldots($ see $(1.21))$, is a positive operator in $\mathscr{H}^{2 n+1}$ for every positive integer $n$.

Moreover, the following statements are equivalent:

(i') $g(z)=\sum_{l=-N}^{N} g_{l} z^{l} \in \mathscr{R}_{0, \infty}^{s}$ is a definitizing function for $F$.

(ii') For $g$ we have $\operatorname{supp}\left(g T_{F}\right) \subset \mathbf{T}$, and $g t_{F}$ is a positive measure on $\mathbf{T}$.

(iv') The numbers $g_{l}, l=-N, \ldots, N$, have the properties mentioned in (iv).

Proof. Assume that (i) holds. Then, by the definition of $T_{F}$ and (2.1), it follows that $\operatorname{supp}\left(g T_{F}\right) \subset \mathbf{T}$. In view of $(1.10), g t_{F}$ is a positive measure.

Let $g \in \mathscr{R}_{0, \infty}^{s}$ and define $K(\lambda):=g T_{F} . f_{\lambda}-(4 \pi)^{-1} g T_{F} . \mathbf{1}, \lambda \in \mathbf{C} \backslash\left(\mathbf{T} \cup \sigma_{0}(F)\right)$, where $f_{\lambda}(z):=(2 \pi)^{-1} z(z-\lambda)^{-1}$. Then

$$
\operatorname{Re} K(\lambda)=\pi\left(g T_{F}\right) \cdot f_{\bar{\lambda}-1} \hat{f}_{\bar{\lambda}-1}|\lambda|^{-2}\left(1-|\lambda|^{2}\right), \quad \lambda \in \mathbf{C} \backslash\left(\mathbf{T} \cup \sigma_{0}(F)\right) .
$$

Moreover (see (1.2)),

$$
\begin{aligned}
K(\lambda) & =g(\lambda)\left(T_{F} \cdot f_{\lambda}\right)+T_{F} \cdot(g-g(\lambda)) f_{\lambda}-(4 \pi)^{-1}\left(g T_{F}\right) \cdot \mathbf{1} \\
& =g(\lambda) F(\lambda)-g(\lambda) F(\infty)+T_{F} \cdot(g-g(\lambda)) f_{\lambda}-(4 \pi)^{-1}\left(g T_{F}\right) .1
\end{aligned}
$$

If (ii) holds, $K$ is holomorphic in $\mathbf{D} \cup \widehat{\mathbf{D}}$ and, by (2.3), $\operatorname{Re} K(\lambda) \geq 0, \lambda \in \mathbf{D}$. Therefore, by $(2.4), F$ belongs to $D_{0}(\mathscr{L}(\mathscr{H}))$ and $g$ is a definitizing function for $F$. Hence (i) and (ii) as well as (i') and (ii') are equivalent.

Let (i) and (i') hold and let $\mu_{1}, \ldots, \mu_{k}$ be the zeros of $g$ on $\mathbf{T}$. Denote the degree of the zero $\mu_{j}, j=1, \ldots, k$, of $g$ by $r_{j}$. Then by (2.1) we have $F \in M_{0}^{m}(\mathscr{L}(\mathscr{H}))$ with $m=\max \left\{r_{j}: j=1, \ldots, k\right\}+1$. Hence $t_{F}$ is an $\mathscr{L}(\mathscr{H})$ valued distribution on $\mathbf{T}$. Then, in view of Lemma 1.7, (iii) is a consequence of (ii). That (iii) implies (ii) can be verified similarly to [9], Hilfssatz 2 . 
Assume again that (ii) holds. Then we have $g T_{F}=T_{K}$ and, on account of Lemma 1.7, $0 \leq T_{K}(u, u) .1, u \in \mathscr{R}_{0, \infty}(\mathscr{H})$. Then the relations (1.23) and (1.22) imply the assertion (iv).

Assume that (iv) holds and set $g(z)=\sum_{l=-N}^{N} g_{l} z^{l}$. Then $T_{F} . f \hat{f} g \geq 0$ for every $f \in \mathscr{R}_{0, \infty}$. Now, approximating the function $f_{\bar{\lambda}-1}$, for arbitrary $\lambda$ with $0<|\lambda|<\varepsilon$, by functions belonging to $\mathscr{R}_{0, \infty}$ we obtain from (2.3) $\operatorname{Re} K(\lambda) \geq 0$ for $|\lambda|<\varepsilon$. Then $K$ can be analytically continued to $\mathbf{D}$ such that $\operatorname{Re} K(\lambda) \geq 0$ for all $\lambda \in \mathbf{D}$, and the relation (2.4) implies (i). Moreover, the above considerations show that $\left(\mathrm{i}^{\prime}\right)$ is equivalent to $\left(\mathrm{iv}^{\prime}\right)$.

Remark 2.3. In the notions of [8] the assertion (iv) means that the sequence $\left(\varphi_{l}\right)$ can be definitized. If (iv) holds, the relations $\varphi_{l}=(2 \pi)^{-1} T_{F} \cdot p_{-l}, l=$ $0, \pm 1, \ldots$, and assertion (ii) yield an integral representation of the sequence $\left(\varphi_{l}\right)$ (cf. also [22]).

Example 2.4. Let $W, U$ and $F_{U}$ be as in Example 1.3 and assume, in addition, that there exists a $g \in \mathscr{R}_{0, \infty}^{s}$ such that

$$
[g(U) x, x] \geq 0, \quad x \in \mathscr{H} .
$$

Then

$$
g(z) F_{U}(z)=G g(U)(U+z I)(U-z I)^{-1}-G(g(U)-g(z) I)(U-z I)^{-1}(U+z I) .
$$

It is easy to see that the first term on the right side of this relation belongs to $C_{0,0}^{+}(\mathscr{L}(\mathscr{H}))$ and that the second term is of the form $i h(z), h \in \mathscr{R}_{0, \infty}^{s}(\mathscr{L}(\mathscr{H}))$. Hence $F_{U} \in D_{0}(\mathscr{L}(\mathscr{H}))$, and $g$ is a definitizing function for $F_{U}$. If $(\mathscr{H},[\cdot, \cdot])$ is a Krein space, unitary operators $U$ in $\mathscr{H}$ with the property (2.5) are called definitizable.

From the well-known operator representations of the functions of the classes $C_{0, \kappa}^{+}(\mathscr{L}(\mathscr{H}))$ it immediately follows, as mentioned in the introduction, that these classes are contained in $D_{0}(\mathscr{L}(\mathscr{H}))$. Now we give a proof of this fact along the lines of [2], proof of Theorem 3.1, where operator representations are not used.

Proposition 2.5. We have $C_{0, \kappa}^{+}(\mathscr{L}(\mathscr{H})) \subset D_{0}(\mathscr{L}(\mathscr{H})), \kappa=0,1, \ldots$ For every $F \in C_{0, \kappa}^{+}(\mathscr{L}(\mathscr{H}))$ there exists a definitizing function $g \in \mathscr{R}_{0, \infty}^{s}$ which is non-negative on $\mathbf{T}$.

Proof. 1. For $\kappa=0$ the assertion follows by the definition of the class $D_{0}(\mathscr{L}(\mathscr{H}))$. Let $\kappa>0$. Assume first that $\mathscr{H}=\mathbf{C}$.

Let $F \in C_{0, \kappa}^{+}(\mathbf{C})$ and let $Q$ denote the set of all functions $q(z)=\sum q_{l} z^{l} \in$ $\mathscr{R}_{0, \infty}^{s}$ such that $q(z) \geq 0$ for $z \in \mathbf{T}$. Let, as above, $\varphi_{i}=(2 \pi)^{-1} T_{F} \cdot p_{-i}, i=$ 
$0, \pm 1, \ldots$ We set

$$
\begin{aligned}
C^{\prime}: & =\left\{\left(\sum_{l} q_{l} \varphi_{-l}, \sum_{l} q_{l} \varphi_{1-l}, \ldots, \sum_{l} q_{l} \varphi_{\kappa-l}\right): q \in Q\right\} \\
& =\left\{\left((2 \pi)^{-1} q T_{F} \cdot p_{0},(2 \pi)^{-1} q T_{F} \cdot p_{-1}, \ldots,(2 \pi)^{-1} q T_{F} \cdot p_{-\kappa}\right): q \in Q\right\} \subset \mathbf{R} \times \mathbf{C}^{\kappa}
\end{aligned}
$$

(see (1.23)). Since every $q \in Q$ can be written as $q=q^{\prime} \widehat{q}^{\prime}, q^{\prime} \in \mathscr{R}_{0, \infty}$, the form $T_{F}(\cdot, \cdot) \cdot q=T_{F}\left(q^{\prime} \cdot, q^{\prime} \cdot\right) .1$ has no more than $\kappa$ negative squares and the Hermitian symmetric Toeplitz matrix $\left(\sum_{l} q_{l} \varphi_{j-k-l}\right)_{j, k=0}^{\kappa}$ has no more than $\kappa$ negative eigenvalues (see (1.22), (1.23)).

We denote by $C^{\prime \prime}$ the set of all vectors $\left(c_{0}^{\prime \prime}, c_{1}^{\prime \prime}, \ldots, c_{\kappa}^{\prime \prime}\right) \in \mathbf{R} \times \mathbf{C}^{\kappa}$ such that the Toeplitz matrix $\left(c_{j-k}^{\prime \prime}\right)_{j, k=0}^{\kappa}, c_{-l}^{\prime \prime}:=\overline{c_{l}^{\prime \prime}}, l=1, \ldots, \kappa$, has only non-negative eigenvalues.

Evidently, $C:=C^{\prime}+C^{\prime \prime}$ is a cone. If $\left(c_{0}, c_{1}, \ldots, c_{\kappa}\right) \in C$, then $\left(c_{j-k}\right)_{j, k=0}^{\kappa}$, $c_{-l}=\overline{c_{l}}, l=1, \ldots, \kappa$, has no more than $\kappa$ negative eigenvalues.

Let $\tilde{c}:=\left(\tilde{c}_{0}, \tilde{c}_{1}, \ldots, \tilde{c}_{\kappa}\right) \in \mathbf{R} \times \mathbf{C}^{\kappa}$ such that $\left(\tilde{c}_{j-k}\right)_{j, k=0}^{\kappa}, \tilde{c}_{-l}=\overline{\tilde{c}_{l}}, l=$ $1, \ldots, \kappa$, has $\kappa+1$ negative eigenvalues. Then $\tilde{c}$ has a positive distance from the cone $C$ in $\mathbf{R} \times \mathbf{C}^{\kappa}$. Hence there exists a vector $b=\left(b_{0}, b_{1}, \ldots, b_{\kappa}\right) \in \mathbf{R} \times \mathbf{C}^{\kappa}$, $b \neq 0$, such that

$$
c_{0} b_{0}+\operatorname{Re} \sum_{j=1}^{\kappa} c_{j} \bar{b}_{j} \geq 0 \quad \text { if }\left(c_{0}, c_{1}, \ldots, c_{\kappa}\right) \in C .
$$

Evidently, $\left(1, e^{-i t}, \ldots, e^{-i \kappa t}\right) \in C$ for every $t \in \mathbf{R}$. Hence, by (2.6)

$$
b_{0}+\sum_{j=1}^{\kappa} \frac{1}{2} b_{j} e^{i j t}+\sum_{j=1}^{\kappa} \frac{1}{2} \bar{b}_{j} e^{-i j t} \geq 0, \quad t \in \mathbf{R},
$$

i.e. the function $g(z):=b_{0}+\sum_{j=1}^{\kappa} \frac{1}{2} b_{j} z^{j}+\sum_{j=1}^{\kappa} \frac{1}{2} \bar{b}_{j} z^{-j}$ is non-negative on $\mathbf{T}$. For arbitrary $q \in Q$ we obtain by (2.6)

$$
0 \leq q T_{F} \cdot b_{0} 1+q T_{F} \cdot \sum_{j=1}^{\kappa} \frac{1}{2} b_{j} p_{j}+q T_{F} \cdot \sum_{j=1}^{\kappa} \frac{1}{2} \overline{b_{j}} p_{-j}=T_{F} . q g .
$$

Then it follows, as in the proof of Proposition $2.2(($ iv $) \Rightarrow(\mathrm{i}))$, that $F \in D_{0}(\mathbf{C})$ and that $g$ is a definitizing function for $F$.

2. Now we consider the general case. Let $F \in C_{0, \infty}^{+}(\mathscr{L}(\mathscr{H}))$. Then $\sigma_{0}(F)$ is finite by Remark 1.6. For arbitrary $x \in \mathscr{H}, z \mapsto(F(z) x, x)$ belongs to 
$\bigcup_{\kappa^{\prime}=0}^{\kappa} C_{0, \kappa^{\prime}}^{+}(\mathbf{C})$ and, by the first part of the proof, there exists a $\delta>0$ (not depending on $x$ ) such that

$$
\sup \left\{|(F(z) x, x)|(1-|z|)^{2 \kappa+1}: 1-\delta<|z|<1\right\}<\infty
$$

Then, by the principle of uniform boundedness, $F \in M_{0}^{\infty}(\mathscr{L}(\mathscr{H}))$.

We assert that there exists a finite set $e \subset \mathbf{T}$ such that all subarcs of $\mathbf{T}$ contained in $\mathbf{T} \backslash e$ are of positive type with respect to $F$. Indeed, suppose that there exists a sequence $\lambda_{i} \in \mathbf{T}, i=1,2, \ldots, \lambda_{i} \neq \lambda_{j}$ for $i \neq j$ such that for every $i$ there exists no open arc $\gamma \ni \lambda_{i}$ of positive type. This implies that $\kappa_{-}(\mathbf{1} ; F)=\infty$, a contradiction. By Proposition 2.2 it follows that $F \in D_{0}(\mathscr{L}(\mathscr{H}))$.

3. To prove the last assertion, we assume that the set $e=\left\{\mu_{1}, \ldots, \mu_{l}\right\}$ is minimal with the above-mentioned properties. Let $\gamma_{j}, j=1, \ldots, l$, and $\chi_{j}$, $j=0, \ldots, l$, be as in Section 1.3 and assume, in addition, that $\mu_{j} \in \gamma_{j}, \mu_{j} \notin \gamma_{k}$, $j, k=1, \ldots, l, j \neq k$. Define $F_{j}, j=0, \ldots, l$, as in Section 1.3. Let $\nu_{1}, \ldots, \nu_{m}$ be the poles of $F$ in $\mathbf{D}$ and let $n_{k}$ be the multiplicity of $\nu_{k}, k=1, \ldots, m$. Then

$$
g_{0}(z):=\prod_{k=1}^{m}\left(z^{-1}-\bar{\nu}_{k}\right)^{n_{k}}\left(z-\nu_{k}\right)^{n_{k}}
$$

is a definitizing function for $F_{0}$ (see the proof of Lemma 1.5), and $\sum_{k=1}^{m} n_{k}=$ $\kappa_{-}\left(\chi_{0} ; F\right)=: \kappa_{0}$ (see Lemma 1.5). The degree of $g_{0}$ is $\kappa_{0}$, and $g_{0}$ is positive on $\mathbf{T}$.

Let $j$ be one of the numbers $1, \ldots, l$. Now we prove that the function $F_{j}$ has a definitizing function of degree

$$
\kappa_{-}\left(1 ; F_{j}\right)=\kappa_{-}\left(\chi_{j} ; F\right)=: \kappa_{j} .
$$

By the first part of the proof, there exists for every $x \in \mathscr{H}$ a function of the form

$$
\prod_{\alpha=1}^{\kappa_{j}}\left(z-z_{\alpha}\right)\left(z^{-1}-\bar{z}_{\alpha}\right), \quad z_{\alpha} \in \mathbf{C},
$$

which is definitizing for $z \mapsto\left(F_{j}(z) x, x\right)$. Then we prove as in [10], proof of Lemma 3 , that

$$
g_{j}(z):=\left(z-\mu_{j}\right)^{\kappa_{j}}\left(z^{-1}-\bar{\mu}_{j}\right)^{\kappa_{j}}
$$

is also definitizing for $z \mapsto\left(F_{j}(z) x, x\right)$. Hence $g_{j}$ is definitizing for $F_{j}$.

It is easy to see that the function

$$
g:=g_{0} g_{1} \cdots g_{l}
$$

is a definitizing function for $F$. Since $\sum_{j=0}^{l} \kappa_{j}=\kappa$, the degree of $g$ is $\kappa$, and Proposition 2.5 is proved. 
The function $g \in \mathscr{R}_{0, \infty}^{s}$ defined by (2.7)-(2.10) will be called the normal definitizing function for $F \in C_{0, \kappa}^{+}(\mathscr{L}(\mathscr{H}))$.

Let $\operatorname{dim} \mathscr{H}<\infty$. Then it is easy to see that for every $g \in \mathscr{R}_{0, \infty}^{s}, \mathscr{R}_{g}:=$ $\left\{g f: f \in \mathscr{R}_{0, \infty}(\mathscr{H})\right\}$ is a linear subspace of $\mathscr{R}_{0, \infty}(\mathscr{H})$ with finite codimension. If, in addition, $g$ is a definitizing function for a function $F \in D_{0}(\mathscr{L}(\mathscr{H}))$ and $g$ is non-negative on $\mathbf{T}, g=h \hat{h}$ with $h \in \mathscr{R}_{0, \infty}$, we have

$$
T_{F}(g f, g f) .1=T_{F}(h f, h f) . g \geq 0, \quad f \in \mathscr{R}_{0, \infty}(\mathscr{H}),
$$

and, hence, $\kappa_{-}(1 ; F)<\infty$. Then, making use of Propositions 2.2 and 2.5 we obtain

Corollary 2.6. Let $\operatorname{dim} \mathscr{H}<\infty$ and $F \in M_{0}^{\infty}(\mathscr{L}(\mathscr{H}))$. Then $F$ belongs to one of the classes $C_{0, \kappa}^{+}(\mathscr{L}(\mathscr{H})), \kappa=0,1, \ldots$, if and only if there exists a finite set $e \subset \mathbf{T}$ such that all subarcs of $\mathbf{T} \backslash e$ are of positive type with respect to $F$.

Analogues of the functionals $T_{F}$ and $T_{F}(\cdot, \cdot)$ with $F$ as in Corollary 2.6 and $\operatorname{dim} \mathscr{H}=1$ were studied in [13] in connection with models of cyclic self-adjoint operators in Pontryagin spaces.

2.2. The critical points. Let $F \in D_{0}(\mathscr{L}(\mathscr{H}))$. A point $\lambda \in \mathbf{T}$ is called a critical point of $F$ if $\lambda$ is not contained in an open arc of positive or of negative type. According to Proposition 2.2, the set of all critical points of $F$, which will be denoted by $c(F)$, is finite. By $c_{\infty}(F)$ we denote the set of those points $\lambda$ of $\mathbf{T}$ which are not contained in an open arc of type $\pi_{+}$or $\pi_{-}$. Let $\sigma_{+}^{\mathbf{D}}(F)$ $\left(\sigma_{-}^{\mathbf{D}}(F)\right)$ be the union of $\sigma_{0}(F) \cap \mathbf{D}$ and the set of all points $\lambda \in \mathbf{T} \cap \sigma(F)$ such that $\lambda$ is not contained in an open arc of $\mathbf{T}$ of negative (positive) type (see Lemma 1.7). Analogously, replacing $\mathbf{D}$ with $\widehat{\mathbf{D}}$, we define the sets $\sigma_{+}^{\widehat{\mathbf{D}}}(F)$ and $\sigma_{-}^{\widehat{\mathbf{D}}}(F)$. Evidently, we have

$$
\sigma(F)=\sigma_{+}^{\mathbf{D}}(F) \cup \sigma_{-}^{\widehat{\mathbf{D}}_{(}}(F), \quad c(F)=\sigma_{+}^{\mathbf{D}}(F) \cap \sigma_{-}^{\widehat{\mathbf{D}}}(F) .
$$

Lemma 2.7. If $\lambda \in \mathbf{T} \cap \sigma(F)$ and there exists a definitizing function $g \in$ $\mathscr{R}_{0, \infty}^{s}$ for $F$ such that $g(\lambda)>0$ (or $g(\lambda)<0$ ), then $\lambda \in \sigma_{+}^{\mathbf{D}}(F) \backslash c(F)(\lambda \in$ $\left.\sigma_{-}^{\mathbf{D}}(F) \backslash c(F)\right)$.

Moreover, there exists a definitizing function $g_{0} \in \mathscr{R}_{0, \infty}^{s}$ for $F$ such that

$$
c(F)=\mathscr{N}\left(g_{0}\right) \cap\left(\sigma(F) \backslash \sigma_{0}(F)\right) \quad \text { and } \quad \sigma_{0}(F)=\mathscr{N}\left(g_{0}\right) \backslash \mathbf{T},
$$

where $\mathscr{N}\left(g_{0}\right):=\left\{z \in \mathbf{C}: g_{0}(z)=0\right\}$.

The first assertion of Lemma 2.7 is a consequence of Proposition 2.2. The second assertion can be proved similarly to [10], Lemma 3 . 
2.3. The spectral function. Let $F \in D_{0}(\mathscr{L}(\mathscr{H}))$. We denote by $\mathfrak{B}(F)$ the Boolean algebra of Borel subsets $b$ of $\overline{\mathbf{C}}$ such that

$$
\partial_{\sigma(F)}(b \cap \sigma(F)) \cap c(F)=\emptyset .
$$

Here the first term is the boundary of the set $b \cap \sigma(F)$ with respect to the relative topology of $\sigma(F)$ (as a subset of $\overline{\mathbf{C}}$ ).

By $B^{\infty}(F, \mathscr{H})$ we denote the linear space of all bounded Borel measurable $\mathscr{H}$-valued functions $f$ defined on the union of $\mathbf{T} \cap \sigma(F)$ and an open neighbourhood $\mathfrak{U}_{0}$ of $\sigma_{0}(F)$ (depending on $f$ ), $\mathfrak{U}_{0} \cap \mathbf{T}=\emptyset$, such that

(i) $\left\{f(z): z \in(\mathbf{T} \cap \sigma(F)) \cup \mathfrak{U}_{0}\right\}$ is contained in some finite-dimensional subspace of $\mathscr{H}$ (depending on $f$ ).

(ii) There exist an open neighbourhood $\mathfrak{U}_{1}$ of $c(F)$ in $\mathbf{T}$ (depending on $f$ ) and a $C^{\infty}$ function $f_{1}$ on $\mathfrak{U}_{1}$ such that $f$ and $f_{1}$ coincide on $\mathfrak{U}_{1} \cap \sigma(F)$.

(iii) $f$ is locally holomorphic on $\mathfrak{U}_{0}$.

Set $B^{\infty}(F):=B^{\infty}(F, \mathbf{C})$.

For an arbitrary $b \in \mathfrak{B}(F)$ we define a function $\tilde{\chi}_{b} \in B^{\infty}(F)$ as follows: We set $\tilde{\chi}_{b}(z)=1$ if $z \in b \cap \mathbf{T} \cap \sigma(F)$ and $\tilde{\chi}_{b}(z)=0$ if $z \in(\mathbf{T} \cap \sigma(F)) \backslash b$. Further, $\tilde{\chi}_{b}$ is equal to 1 in some neighbourhood of $b \cap \sigma_{0}(F)$ and equal to 0 in some neighbourhood of $\sigma_{0}(F) \backslash b$.

The functionals $T_{F}$ and $T_{F}(\cdot, \cdot)$ can be extended by continuity to $B^{\infty}(F)$ and $B^{\infty}(F, \mathscr{H}) \times B^{\infty}(F, \mathscr{H}) \times B^{\infty}(F)$, respectively. It is sufficient to verify this for functions $f_{0} \in B^{\infty}(F)$ and $u_{0}, v_{0} \in B^{\infty}(F, \mathscr{H})$ which are zero on some neighbourhood of $c(F)$ in $\mathbf{T} \cap \sigma(F)$. Let $\gamma_{0}$ and $\gamma$ be open subsets of $\mathbf{T} \backslash c(F)$ such that $\bar{\gamma}_{0} \subset \gamma, \bar{\gamma} \subset \mathbf{T} \backslash c(F)$ and $f_{0}, u_{0}$ and $v_{0}$ are zero on $(\mathbf{T} \cap \sigma(F)) \backslash \gamma_{0}$. Let the functions $f_{n} \in C^{\infty}(\mathbf{T}) \times H\left(\sigma_{0}(F)\right), u_{n}, v_{n} \in C^{\infty}(\mathbf{T}, \mathscr{H}) \times H\left(\sigma_{0}(F), \mathscr{H}\right)$, $n=1,2, \ldots$, with supports contained in $\gamma$ have the following properties:

(a) $f_{n}, u_{n}, v_{n}, n=1,2, \ldots$, are uniformly bounded.

(b) $\bigcup_{n}\left\{u_{n}(z): z \in \mathbf{T}\right\} \cup\left\{v_{n}(z): z \in \mathbf{T}\right\}$ is contained in some finitedimensional subspace of $\mathscr{H}$.

(c) The sequences $\left(f_{n}\right),\left(u_{n}\right),\left(v_{n}\right)$ converge pointwise to some functions $f$, $u, v$, respectively.

Then by Lemma 1.7 ((i) $\Rightarrow$ (ii)), the sequences

$$
\left(T_{F} \cdot f_{n}\right) \quad \text { and } \quad\left(T_{F}\left(u_{n}, v_{n}\right) \cdot f_{n}\right)
$$

converge weakly. We denote the limits by $T_{F} . f$ and $T_{F}(u, v) . f$, respectively. Repeating this procedure (transfinite induction) we define $T_{F} \cdot f_{0}$ and $T_{F}\left(u_{0}, v_{0}\right) \cdot f_{0}$ (cf. [10], p. 127).

We now define

$$
\begin{aligned}
E_{F}(b) & :=T_{F} \cdot \tilde{\chi}_{b}, \quad b \in \mathfrak{B}(F), \\
E_{F}(u, v ; b) & :=T_{F}(u, v) \cdot \tilde{\chi}_{b}, \quad u, v \in B^{\infty}(F, \mathscr{H}), b \in \mathfrak{B}(F) .
\end{aligned}
$$


These set functions on $\mathfrak{B}(F)$ are called the spectral function and the form spectral function of $F$, respectively.

If $b$ is $\mathbf{T}$-symmetric, $E_{F}(b)$ is self-adjoint and $E_{F}(\cdot, \cdot ; b)$ is a Hermitian sesquilinear form. The restriction of $E_{F}(\cdot)$ to $\mathbf{T} \backslash c(F)$ is an $\mathscr{L}(\mathscr{H})$-valued (generally unbounded) measure. If $\gamma \subset \mathbf{T}$ is an open arc of positive (negative) type and $b$ is a relatively compact Borel subset of $\gamma$, then $E_{F}(b) \geq 0\left(E_{F}(b) \leq 0\right)$ and $E_{F}(\cdot, \cdot ; b)$ is a positive semidefinite (negative semidefinite) form on $B^{\infty}(F, \mathscr{H})$ (or, equivalently, on $\mathscr{R}_{0, \infty}(\mathscr{H})$ ).

For every $b \in \mathfrak{B}(F), b \subset \overline{\mathbf{D}}$, we define

$$
\left\{\begin{array}{l}
r_{+}^{\mathbf{D}}(b ; F):=\kappa_{+}\left(\left(\mathscr{R}_{0, \infty}(\mathscr{H}), E_{F}(\cdot, \cdot ; b \cup \hat{b})\right)\right), \\
r_{-}^{\mathbf{D}}(b ; F):=\kappa_{-}\left(\left(\mathscr{R}_{0, \infty}(\mathscr{H}), E_{F}(\cdot, \cdot ; b \cup \hat{b})\right)\right) .
\end{array}\right.
$$

If $\delta$ is an arbitrary subset of $\overline{\mathbf{D}}$ which is open in $\overline{\mathbf{D}}$ we set

$$
\left\{\begin{array}{l}
r_{+}^{\mathbf{D}}(\delta ; F):=\sup \left\{r_{+}^{\mathbf{D}}(b ; F): b \in \mathfrak{B}(F), b \subset \delta\right\}, \\
r_{-}^{\mathbf{D}}(\delta ; F):=\sup \left\{r_{-}^{\mathbf{D}}(b ; F): b \in \mathfrak{B}(F), b \subset \delta\right\} .
\end{array}\right.
$$

It is easy to see that we have $\sigma_{ \pm}^{\mathbf{D}}(F) \cap \delta=\emptyset$ if and only if $r_{ \pm}^{\mathbf{D}}(\delta ; F)=0$. If $\sigma_{ \pm}^{\mathbf{D}}(F) \cap \delta$ is an infinite set, we have $r_{ \pm}^{\mathbf{D}}(\delta ; F)=\infty$. For every $\lambda \in \sigma_{ \pm}^{\mathbf{D}}(F)$ the quantity

$$
\inf \left\{r_{ \pm}^{\mathbf{D}}(\delta ; F): \delta \subset \overline{\mathbf{D}}, \delta \text { open in } \overline{\mathbf{D}}, \lambda \in \delta\right\}
$$

is called the multiplicity of $\lambda$ (for the notation cf. Example 2.9).

In the following lemma we express the quantities (2.12) as limits of the ranks of positivity and negativity which were introduced in Section 1.2. For an open $\mathbf{T}$-symmetric subset $G$ of $\overline{\mathbf{C}}$ and $\varepsilon \in(0,1)$, let $\Xi(G, \varepsilon)$ denote the set of all real $C^{\infty}$ functions $\chi$ on $\overline{\mathbf{C}}$ with the following properties:

$(\alpha) \chi(z)=1$ if $\operatorname{dist}_{\overline{\mathbf{C}}}(z, \overline{\mathbf{C}} \backslash G) \geq \varepsilon, \chi(z)=0$ if $\operatorname{dist}_{\overline{\mathbf{C}}}(z, \overline{\mathbf{C}} \backslash G) \leq \frac{1}{2} \varepsilon$, where $\operatorname{dist}_{\overline{\mathbf{C}}}(\cdot, \cdot)$ is the distance on the Riemann complex sphere.

( $\beta) 0 \leq \chi(z) \leq 1, z \in \overline{\mathbf{C}}$.

$(\gamma) \chi(z)=\chi\left(\bar{z}^{-1}\right), z \in \overline{\mathbf{C}}$.

$(\delta) \chi^{1 / 2}$ and $(1-\chi)^{1 / 2}$ are $C^{\infty}$ functions.

Lemma 2.8. Let the set $\delta \subset \overline{\mathbf{D}}$ be open in $\overline{\mathbf{D}}$, let $\varepsilon_{n} \in(0,1), n=1,2, \ldots$, $\varepsilon_{n} \downarrow 0$, and let $\chi_{n} \in \Xi\left(\delta \cup \hat{\delta}, \varepsilon_{n}\right), n=1,2, \ldots$ Then $\kappa_{ \pm}\left(\chi_{n} ; F\right)$ is defined for sufficiently large $n$ and

$$
r_{ \pm}^{\mathbf{D}}(\delta ; F)=\sup _{n} \kappa_{ \pm}\left(\chi_{n} ; F\right) .
$$

Proof. By the definition of the spectral function we have $r_{ \pm}^{\mathbf{D}}(\delta ; F) \leq$ $\sup _{n} \kappa_{ \pm}\left(\chi_{n} ; F\right)$. Since, for every $u, v \in \mathscr{R}_{0, \infty}(\mathscr{H})$,

$$
T_{F}(u, v) \cdot \chi_{n}=T_{F}\left(\chi_{n}^{1 / 2} u, \chi_{n}^{1 / 2} v\right) .1=E_{F}\left(\chi_{n}^{1 / 2} u, \chi_{n}^{1 / 2} v ; G\right), \quad n=1,2, \ldots,
$$

the opposite inequality is also true. 
Example 2.9. Let $[\cdot, \cdot], U$ and $F_{U}$ be as in Example 1.3 and assume, in addition, that $(\mathscr{H},[\cdot, \cdot])$ is a Krein space, $[\cdot, \cdot]=(G \cdot, \cdot)$ and $U$ is definitizable in $(\mathscr{H},[\cdot, \cdot])$. Then, according to Example 1.4, we have $c(U)=c\left(F_{U}\right)$. Furthermore,

$$
4 \pi G E(b ; U)=E_{F_{U}}(b), \quad b \in \mathfrak{B}\left(F_{U}\right),
$$

where $E(\cdot ; U)$ is the spectral function of $U$ (see e.g. [11], Section 2.2).

For every set $b \subset \overline{\mathbf{D}}, b \in \mathfrak{B}\left(F_{U}\right)$, which is open in $\overline{\mathbf{D}}$, we have

$$
r_{ \pm}^{\mathbf{D}}\left(b ; F_{U}\right)=\kappa_{ \pm}((\mathscr{H},[E(b \cup \hat{b} ; U) \cdot, \cdot])) .
$$

This is a consequence of (1.16) and Lemma 2.7.

Let $\mathscr{M}_{+}$and $\mathscr{M}_{-}$be as in the introduction. Then it is easy to see that

$$
\sigma_{ \pm}^{\mathbf{D}}\left(F_{U}\right)=\sigma\left(U \mid \mathscr{M}_{ \pm}\right)
$$

For $b$ as in (2.15) let $\sigma\left(U \mid \mathscr{M}_{ \pm}\right) \cap b$ be a finite set. Then $r_{ \pm}^{\mathrm{D}}\left(b ; F_{U}\right)$ is the total algebraic multiplicity of the points of the spectrum of $U \mid \mathscr{M}_{ \pm}$in $b$ (cf. [12], Proposition 2.7).

\section{Convergent sequences of functions of the class $D_{0}(\mathscr{L}(\mathscr{H}))$}

Let the functions $F_{n} \in D_{0}(\mathscr{L}(\mathscr{H})), n=1,2, \ldots$, and $F_{\infty} \in M_{0}(\mathscr{L}(\mathscr{H}))$ satisfy the following conditions:

(i) There exists an $\eta \in(0,1)$ such that

$$
\sigma\left(F_{n}\right) \cap\{z:|z| \leq \eta\}=\emptyset, \quad n=1,2, \ldots, \infty .
$$

(ii) For every pair $x, y \in \mathscr{H}$ we have

$$
\lim _{n \rightarrow \infty}\left(F_{n}(z) x, y\right)=\left(F_{\infty}(z) x, y\right)
$$

uniformly in $\{z:|z| \leq \eta\}$.

(iii) There is a positive integer $N$ and a sequence of functions $g_{n} \in \mathscr{R}_{0, \infty}^{s}$, $n=1,2, \ldots$, of degree $\leq N$ such that $g_{n}$ is a definitizing function of $F_{n}, n=$ $1,2, \ldots$

In this section we consider the distribution sequence $\left(T_{F_{n}}\right)$, the set sequences $\left(\sigma_{+}^{\mathbf{D}}\left(F_{n}\right)\right)$ and $\left(\sigma_{-}^{\mathbf{D}}\left(F_{n}\right)\right)$, and the corresponding sequences of multiplicity functions. 
Theorem 3.1. Let $F_{n} \in D_{0}(\mathscr{L}(\mathscr{H})), n=1,2, \ldots, F_{\infty} \in M_{0}(\mathscr{L}(\mathscr{H}))$, and assume the above conditions (i), (ii), (iii) to be satisfied. Then the following holds:

(1) $F_{\infty} \in D_{0}(\mathscr{L}(\mathscr{H}))$.

(2) For every pair $x, y \in \mathscr{H}$ and every $f \in \mathscr{R}_{0, \infty}$ we have

$$
\lim _{n \rightarrow \infty}\left(T_{F_{n}} \cdot f x, y\right)=\left(T_{F_{\infty}} \cdot f x, y\right) .
$$

(3) For every set $\delta_{0} \subset \overline{\mathbf{D}}$ which is open in $\overline{\mathbf{D}}$ we have

$$
r_{+}^{\mathbf{D}}\left(\delta_{0} ; F_{\infty}\right) \leq \liminf _{n \rightarrow \infty} r_{+}^{\mathbf{D}}\left(\delta_{0} ; F_{n}\right), \quad r_{-}^{\mathbf{D}}\left(\delta_{0} ; F_{\infty}\right) \leq \liminf _{n \rightarrow \infty} r_{-}^{\mathbf{D}}\left(\delta_{0} ; F_{n}\right) .
$$

Proof. The assertion (2) is a consequence of (1.21). To prove (3) it is sufficient to show that every subsequence $\left(F_{n}^{0}\right)$ of $\left(F_{n}\right)$ possesses a subsequence $\left(F_{n}^{00}\right)$ for which (3) holds. Every sequence of functions of $\mathscr{R}_{0, \infty}^{s}$ of degree $\leq N$ possesses a subsequence such that the corresponding $(2 N+1)$-systems of coefficients converge, which is equivalent to the uniform convergence of that subsequence on every compact subset of $\mathbf{C} \backslash\{0\}$. Hence it is sufficient to verify (3) under the additional assumption that

$$
\lim _{n \rightarrow \infty} g_{n}(z)=: g_{\infty}(z)
$$

exists uniformly on all compact subsets of $\mathbf{C} \backslash\{0\}$. We have $g_{\infty} \in \mathscr{R}_{0, \infty}^{s}$.

We define holomorphic functions $H_{n}$ in $\mathbf{D}$ with $\operatorname{Re} H_{n}(z) \geq 0, z \in \mathbf{D}$, and $\operatorname{Im} H_{n}(0)=0$ and functions $h_{n} \in \mathscr{R}_{0, \infty}^{s}(\mathscr{L}(\mathscr{H}))$ by

$$
g_{n}(z) F_{n}(z)=H_{n}(z)+i h_{n}(z), \quad z \in \mathbf{D}, n=1,2, \ldots
$$

Then by (ii) and (2.2) there exists an $h_{\infty} \in \mathscr{R}_{0, \infty}^{s}(\mathscr{L}(\mathscr{H}))$ such that for every pair $x, y \in \mathscr{H}$ we have

$$
\lim _{n \rightarrow \infty}\left(h_{n}(z) x, y\right)=\left(h_{\infty}(z) x, y\right)
$$

uniformly on every compact subset of $\mathbf{C} \backslash\{0\}$. Hence, for every $x, y \in \mathscr{H}$, we have

$$
\lim _{n \rightarrow \infty}\left(H_{n}(z) x, y\right)=\left(H_{\infty}(z) x, y\right)
$$

uniformly in $\{z:|z| \leq \eta\}$, where $H_{\infty}$ is a function belonging to the class $C_{0,0}^{+}(\mathscr{L}(\mathscr{H}))$. Since

$$
g_{\infty}(z) F_{\infty}(z)=H_{\infty}(z)+i h_{\infty}(z), \quad z \in \mathbf{D}
$$


we conclude that assertion (1) holds, and $g_{\infty}$ is a definitizing function for $F_{\infty}$.

Now we verify as in [6], Lemma II.3, that for every fixed $x \in \mathscr{H}$ the functions $\left(H_{n}(z) x, x\right), n=1,2, \ldots$, are uniformly bounded and equicontinuous on every compact subset of $\mathbf{D}$. Hence every subsequence of this sequence contains a subsequence which converges uniformly on every compact subset of $\mathbf{D}$. Since, on the other hand, the limits of all such subsequences coincide with $H_{\infty}$ by (3.4), it follows that for every $x, y \in \mathscr{H}$ we have

$$
\lim _{n \rightarrow \infty}\left(H_{n}(z) x, y\right)=\left(H_{\infty}(z) x, y\right), \quad z \in \mathbf{D},
$$

uniformly on all compact subsets of $\mathbf{D}$. Moreover, there exists a constant $M$ such that

$$
\left\|H_{n}(z)\right\| \leq\left. M|1-| z\right|^{-1}, \quad z \in \mathbf{D}, n=1,2, \ldots, \infty
$$

(see [1], Section 2).

In assertion (3) the case of a general set $\delta_{0} \subset \overline{\mathbf{D}}$ which is open in $\overline{\mathbf{D}}$ can easily be reduced to the following cases:

(a) $\delta_{0}=\left\{z: \operatorname{dist}_{\overline{\mathbf{C}}}\left(z, z_{0}\right)<\varepsilon_{0}\right\}$, where $z_{0} \in \sigma_{0}\left(F_{\infty}\right)$ and $\varepsilon_{0}$ is chosen such that $\bar{\delta}_{0} \cap\left(\sigma_{0}\left(F_{\infty}\right) \cup \mathbf{T}\right)=\left\{z_{0}\right\}$.

(b) $\delta_{0}=\left\{z \in \overline{\mathbf{D}}: \operatorname{dist}_{\overline{\mathbf{C}}}(z, \gamma)<\varepsilon_{0}\right\}$, where $\gamma$ is a closed arc of $\mathbf{T}$ and $\varepsilon_{0}$ is chosen such that $\bar{\delta}_{0} \cap \sigma_{0}\left(F_{\infty}\right)=\emptyset$.

Assume first that (a) holds. We assume, in addition, that the boundary of $\delta_{0} \cup \hat{\delta}_{0}$ contains no zero of $g_{\infty}$. This is no restriction. According to (3.1) there exists an integer $n_{0}$ such that $\bigcup\left\{\mathscr{N}\left(g_{n}\right): n \geq n_{0}\right\}$ has a positive distance from the boundary of $\delta_{0} \cup \hat{\delta}_{0}$. Then by (3.1), (3.3) and (3.5) we obtain

$$
\lim _{n \rightarrow \infty} E_{F_{n}}\left(u, v ; \delta_{0} \cup \hat{\delta}_{0}\right)=E_{F_{\infty}}\left(u, v ; \delta_{0} \cup \hat{\delta}_{0}\right), \quad u, v \in \mathscr{R}_{0, \infty}(\mathscr{H}),
$$

which implies (3).

Let now the condition (b) be satisfied. We may further assume that

$$
\delta_{0} \cap \mathscr{N}\left(g_{\infty}\right) \subset \gamma .
$$

If $r_{+}^{\mathbf{D}}\left(\delta_{0} ; F_{\infty}\right)<\infty$, we set $r_{0}:=r_{+}^{\mathbf{D}}\left(\delta_{0} ; F_{\infty}\right)$. If $r_{+}^{\mathbf{D}}\left(\delta_{0} ; F_{\infty}\right)=\infty, r_{0}$ is assumed to be an arbitrary positive integer. Then, by Lemma 2.7 , we find a function $\chi \in \Xi\left(\delta_{0} \cup \hat{\delta}_{0}, \varepsilon_{0}^{\prime}\right), \varepsilon_{0}^{\prime} \in\left(0, \varepsilon_{0}\right)$, such that

$$
\kappa_{+}\left(\chi ; F_{\infty}\right) \geq r_{0}
$$

By (3.1) and (3.7) there exists an integer $n_{0}$ such that $\bigcup\left\{\mathscr{N}\left(g_{n}\right): n \geq n_{0}\right\}$ has a positive distance from

$$
S:=\left\{z: \frac{1}{2} \varepsilon_{0}^{\prime} \leq \operatorname{dist}_{\overline{\mathbf{C}}}\left(z, \overline{\mathbf{C}} \backslash\left(\delta_{0} \cup \hat{\delta}_{0}\right)\right) \leq \varepsilon_{0}^{\prime}\right\} .
$$


Then by (3.6) there exists a constant $M^{\prime}$ such that

$$
\left\|F_{n}(z)\right\| \leq M^{\prime}|1-| z \|^{-1}, \quad z \in S \backslash \mathbf{T}, n \geq n_{0} .
$$

Making use of this relation and the relations (3.1), (3.3) and (3.5), one verifies similarly to the proof of Proposition 1.2 (see also [11], Proposition 1.1) that

$$
\lim _{n \rightarrow \infty} T_{F_{n}}(u, v) \cdot \chi=T_{F_{\infty}}(u, v) \cdot \chi
$$

for every pair $u, v \in \mathscr{R}_{0, \infty}(\mathscr{H})$. In view of $(3.8)$ it follows that $\kappa_{+}\left(\chi ; F_{n}\right) \geq r_{0}$ for sufficiently large $n$. By Lemma 2.7 this implies the first relation of (3). The second relation of (3) can be proved in a similar way.

If we assume, in addition, that the functions $F_{n}, n=1,2, \ldots, \infty$, belong to a fixed class $C_{0, \kappa}^{+}(\mathscr{L}(\mathscr{H}))$, we get stronger results. The following corollary shows in particular that in this case the sequence $\left(\sigma_{-}^{\mathbf{D}}\left(F_{n}\right)\right)$ converges to $\sigma_{-}^{\mathbf{D}}\left(F_{\infty}\right)$.

Corollary 3.2. Let $F_{n} \in C_{0, \kappa}^{+}(\mathscr{L}(\mathscr{H})), n=1,2, \ldots, \infty$, and assume that the conditions (i) and (ii) are satisfied. Then

(1') $\lim _{n \rightarrow \infty} \sigma_{-}^{\mathbf{D}}\left(F_{n}\right)=\sigma_{-}^{\mathbf{D}}\left(F_{\infty}\right)$ in the sense of the Hausdorff distance in $\overline{\mathbf{C}}$.

$\left(2^{\prime}\right)$ Let the sets $b_{1}, b_{2} \subset \overline{\mathbf{D}}$ be open in $\overline{\mathbf{D}}$ and $\bar{b}_{1} \subset b_{2}$. Then we have

$$
r_{-}^{\mathbf{D}}\left(b_{1} ; F_{\infty}\right) \leq r_{-}^{\mathbf{D}}\left(b_{1} ; F_{n}\right) \leq r_{-}^{\mathbf{D}}\left(b_{2} ; F_{\infty}\right)
$$

for sufficiently large $n$.

$\left(3^{\prime}\right)$ If $f \in C^{\infty}(\mathbf{T}) \times H\left(\sigma_{0}\left(F_{\infty}\right)\right)$ and $f$ is holomorphic in a neighbourhood of $\sigma_{-}^{\mathbf{D}}\left(F_{\infty}\right)$, then

$$
\lim _{n \rightarrow \infty}\left(T_{F_{n}} \cdot f x, y\right)=\left(T_{F_{\infty}} \cdot f x, y\right), \quad x, y \in \mathscr{H} .
$$

$\left(4^{\prime}\right)$ Let $g_{n}$ be the normal definitizing function of $F_{n}, n=1,2, \ldots, \infty$ (see the remark following Proposition 2.5). Then

$$
\lim _{n \rightarrow \infty} g_{n}(z)=g(z)
$$

uniformly on every compact subset of $\mathbf{C} \backslash\{0\}$. For every $x \in \mathscr{H}$ the sequence of the positive measures $\left(g_{n} T_{F_{n}} \cdot(\cdot) x, x\right), n=1,2, \ldots$, converges to the positive measure $\left(g_{\infty} T_{F_{\infty}} \cdot(\cdot) x, x\right)$ with respect to the weak ${ }^{*}$ topology of $(C(\mathbf{T}))^{\prime}$.

$\left(5^{\prime}\right)$ Let $\gamma$ be an arc of $\mathbf{T}$ and $\alpha$ and $\beta$ its endpoints. Assume that $\alpha$ and $\beta$ do not belong to $\sigma_{-}^{\mathbf{D}}\left(F_{\infty}\right)$ and that $E_{F_{\infty}}(\{\alpha\})=E_{F_{\infty}}(\{\beta\})=0$ holds. Then we have

$$
\lim _{n \rightarrow \infty}\left(E_{F_{n}}(\gamma) x, y\right)=\left(E_{F_{\infty}}(\gamma) x, y\right), \quad x, y \in \mathscr{H}
$$


Proof. In view of Proposition 2.5, the functions $F_{n}, n=1,2, \ldots, \infty$, satisfy the condition (iii) with $N=\kappa$.

Let $\sigma_{-}^{\mathbf{D}}\left(F_{\infty}\right)=\left\{\nu_{1}, \ldots, \nu_{m}\right\}$. We choose $\varepsilon_{0}>0$ such that the closures of the sets $\delta_{j}\left(\varepsilon_{0}\right):=\left\{z \in \overline{\mathbf{D}}: \operatorname{dist}_{\overline{\mathbf{C}}}\left(z, \nu_{j}\right)<\varepsilon_{0}\right\}, j=1, \ldots, m$, are pairwise disjoint and for every $\nu_{k}$ with $\nu_{k} \in \mathbf{D}$ we have $\overline{\delta_{k}\left(\varepsilon_{0}\right)} \cap \mathbf{T}=\emptyset$. By Theorem 3.1 there is a positive integer $N_{0}$ such that for $n \geq N_{0}$ we have

$$
r_{-}^{\mathbf{D}}\left(\delta_{j}\left(\varepsilon_{0}\right) ; F_{\infty}\right) \leq r_{-}^{\mathbf{D}}\left(\delta_{j}\left(\varepsilon_{0}\right) ; F_{n}\right), \quad j=1, \ldots, m,
$$

and, hence,

$$
\sum_{j=1}^{m} r_{-}^{\mathbf{D}}\left(\delta_{j}\left(\varepsilon_{0}\right) ; F_{\infty}\right) \leq \sum_{j=1}^{m} r_{-}^{\mathbf{D}}\left(\delta_{j}\left(\varepsilon_{0}\right) ; F_{n}\right) .
$$

Since the left-hand side of this relation is equal to $\kappa$ and the right-hand side is $\leq \kappa$, we get

$$
r_{-}^{\mathbf{D}}\left(\delta_{j}\left(\varepsilon_{0}\right) ; F_{\infty}\right)=r_{-}^{\mathbf{D}}\left(\delta_{j}\left(\varepsilon_{0}\right) ; F_{n}\right), \quad j=1, \ldots, m,
$$

for $n \geq N_{0}$.

Let $b_{1}$ and $b_{2}$ be as in assertion $\left(2^{\prime}\right)$. It is easy to see that (3.9) implies the first inequality in $\left(2^{\prime}\right)$. Let the set $b_{3} \subset \overline{\mathbf{D}}$ be open in $\overline{\mathbf{D}}$ such that $b_{3} \supset \overline{\mathbf{D}} \backslash b_{2}$ and $\bar{b}_{1} \cap \bar{b}_{3}=\emptyset$. Then

$$
\kappa-r_{-}^{\mathbf{D}}\left(b_{2} ; F_{\infty}\right) \leq r_{-}^{\mathbf{D}}\left(b_{3} ; F_{\infty}\right) \leq r_{-}^{\mathbf{D}}\left(b_{3} ; F_{n}\right) \leq \kappa-r_{-}^{\mathbf{D}}\left(b_{1} ; F_{n}\right)
$$

for sufficiently large $n$, which proves the second inequality of $\left(2^{\prime}\right)$. The assertion $\left(1^{\prime}\right)$ is a consequence of $\left(2^{\prime}\right)$.

From $\left(1^{\prime}\right)$ and $\left(2^{\prime}\right)$ it follows that, for the normal definitizing functions $g_{n}$ of $F_{n}, n=1,2, \ldots, \infty$, we have

$$
\lim _{n \rightarrow \infty} g_{n}(z)=g_{\infty}(z)
$$

uniformly on every compact subset of $\mathbf{C} \backslash\{0\}$. We now define a sequence of functions $H_{n}, n=1,2, \ldots, \infty$, as in (3.2). These functions fulfil the relations (3.5) and (3.6). Then one verifies the assertion ( $\left.3^{\prime}\right)$ similarly to [11], Proposition 1.1, making use of (3.10), (3.3), (3.5) and (3.6).

On account of (3.5) the positive measures $g_{n} T_{F_{n}}$ on $\mathbf{T}$ are uniformly bounded. Let $x$ be an arbitrary element of $\mathscr{H}$. Then, by the Helly theorem, every subsequence of $\left(\left(g_{n} T_{F_{n}}\right) \cdot(\cdot) x, x\right)$ contains a subsequence $\left(\left(g_{n_{i}} T_{F_{n_{i}}}\right) \cdot(\cdot) x, x\right)$ which converges in the weak ${ }^{*}$ sense. Since, on account of $\left(3^{\prime}\right)$,

$$
\lim _{i \rightarrow \infty}\left(\left(g_{n_{i}} T_{F_{n_{i}}}\right) \cdot \varphi x, x\right)=\left(\left(g_{\infty} T_{F_{\infty}}\right) \cdot \varphi x, x\right)
$$

for $\varphi \in \mathscr{R}_{0, \infty},\left(\left(g_{n_{i}} T_{F_{n_{i}}}\right) \cdot(\cdot) x, x\right)$ converges to $\left(\left(g_{\infty} T_{F_{\infty}}\right) \cdot(\cdot) x, x\right)$. It follows that $\left(\left(g_{n} T_{F_{n}}\right) \cdot(\cdot) x, x\right)$ converges to $\left(\left(g_{\infty} T_{F_{\infty}}\right) \cdot(\cdot) x, x\right)$ in the weak ${ }^{*}$ sense.

The assertion $\left(5^{\prime}\right)$ is a consequence of $\left(3^{\prime}\right)$ and $\left(4^{\prime}\right)$. 
Remark 3.3. Let the assumptions of Example 1.3 be fulfilled and let, in addition, $(\mathscr{H},[\cdot, \cdot])$ be a Pontryagin space. If, then, the functions $F_{n}$ in Corollary 3.2 have the form (1.14), this corollary can be regarded as a perturbation result for unitary operators in Pontryagin spaces. For a similar result see [21], Theorem 3.4.

Remark 3.4. If, in addition to the assumptions of Theorem 3.1, we have $g_{n}(z) F_{n}(z)=E_{n}(z)+i e_{n}(z), n=1,2, \ldots$, where $E_{n} \in C_{0, \kappa}^{+}(\mathscr{L}(\mathscr{H}))$ for some fixed $\kappa>0$, and $e_{n} \in \mathscr{R}_{0, \infty}^{s}(\mathscr{L}(\mathscr{H}))$, then Corollary 3.2 applied to $E_{n}$ gives some additional information on the sequences $\left(\sigma_{-}^{\mathbf{D}}\left(F_{n}\right)\right)$ and $\left(\sigma_{+}^{\mathbf{D}}\left(F_{n}\right)\right)$.

As an application of Corollary 3.2 we shall show in the following proposition that, for an arbitrary $F \in C_{0, \kappa}^{+}(\mathscr{L}(\mathscr{H}))$, the set $\sigma_{-}^{\mathbf{D}}(F) \cap \mathbf{T}$ coincides with the set of generalized poles of negative type of $F$, which were introduced in [19]. We recall the definition from [19]: A point $\mu \in \mathbf{T}$ is called a generalized pole of negative type and multiplicity $m$ of $F$ if for each sufficiently small $\varepsilon>0$ there exists a number $N(\varepsilon)>0$ such that for $\alpha>N(\varepsilon)$ the function $z \mapsto F(z)+\alpha I$ has zeros of total multiplicity $m$ in $\left\{z \in \mathbf{D}: \operatorname{dist}_{\overline{\mathbf{C}}}(z, \mu)<\varepsilon\right\}$.

Proposition 3.5. Let $F \in C_{0, \kappa}^{+}(\mathscr{L}(\mathscr{H}))$. Then $\sigma_{-}^{\mathbf{D}}(F) \cap \mathbf{T}$ coincides with the set of generalized poles of negative type of $F$. If $\mu \in \sigma_{-}^{\mathbf{D}}(F) \cap \mathbf{T}$, the multiplicity of $\mu$ as an element of $\sigma_{-}^{\mathbf{D}}(F)$ (see (2.13)) coincides with the multiplicity of $\mu$ as a generalized pole of negative type.

Proof. 1. Let $M$ be a positive number such that for every $\alpha>M$ the function $z \mapsto F(z)+\alpha I$ is boundedly invertible in some open neighbourhood $\mathfrak{U}_{\alpha}$ of 0 . Let $\alpha>M$. Then the function

$$
z \mapsto(F(z)-\alpha I)(F(z)+\alpha I)^{-1}=I-2 \alpha(F(z)+\alpha I)^{-1}
$$

can be extrapolated in a unique way to a meromorphic function $\Theta_{\alpha}$ of the class $S_{0, \kappa}^{+}(\mathscr{L}(\mathscr{H}))$, i.e. the kernel $(1-\bar{\zeta} z)^{-1}\left(I-Q_{\alpha}(\zeta)^{*} Q_{\alpha}(z)\right)$ has $\kappa$ negative squares ([16]). Hence $F(z)+\alpha I$ is boundedly invertible for all $z \in \mathbf{D}$ with the exception of a finite number of points and

$$
z \mapsto(F(z)+\alpha I)^{-1}
$$

is meromorphic in D. On account of [16], Satz 3.2, (3.11) has poles in $\mathbf{D}$ of total multiplicity $\kappa$. Hence, in view of Lemma 1.5 , we have $\kappa \leq \kappa_{-}\left(1 ;\left(F^{(\alpha)}\right)^{-1}\right)=$ $\kappa_{-}\left(\mathbf{1} ; F^{(\alpha)}\right)$ for the function $F^{(\alpha)}$, defined by

$$
F^{(\alpha)}(z):= \begin{cases}F(z)+\alpha I & \text { if } z \in \mathbf{D} \\ F(z)-\alpha I & \text { if } z \in \widehat{\mathbf{D}}\end{cases}
$$


On the other hand, by the definition of $T_{F^{(\alpha)}}(\cdot, \cdot) .1$ we have $\kappa_{-}\left(1 ; F^{(\alpha)}\right) \leq \kappa$ and, hence

$$
F^{(\alpha)} \in C_{0, \kappa}^{+}(\mathscr{L}(\mathscr{H}))
$$

A point $\nu \in \mathbf{D}$ is a pole of $F$ if and only if it is a pole of $F^{(\alpha)}$. The multiplicities of $\nu$ with respect to $F$ and $F^{(\alpha)}$ coincide. For every set $\delta \in \overline{\mathbf{D}}$ with $\delta \cap \sigma_{0}(F)=\emptyset$ which is open in $\overline{\mathbf{D}}$ it follows from Lemma 2.7 that $r_{-}^{\mathbf{D}}\left(\delta ; F^{(\alpha)}\right) \leq r_{-}^{\mathbf{D}}(\delta ; F)$. Then, on account of (3.12), we find that $\sigma_{-}^{\mathbf{D}}(F)=\sigma_{-}^{\mathbf{D}}\left(F^{(\alpha)}\right)$ and that, for every $\mu \in \sigma_{-}^{\mathbf{D}}(F) \cap \mathbf{T}$, the multiplicities of $\mu$ as a point of $\sigma_{-}^{\mathbf{D}}(F)$ and as a point of $\sigma_{-}^{\mathbf{D}}\left(F^{(\alpha)}\right)$ coincide.

2. Let $\beta>0$. It is easy to see that

$$
\left(\left(F^{(\alpha)}(z)\right)^{-1}+\beta^{-1}\right)^{-1}=\beta-\beta^{2}\left(F^{(\alpha+\beta)}(z)\right)^{-1}
$$

holds for all $z \in \mathbf{D}$ with the exception of a finite set. As in the first part of the proof we see that the function (3.13) has poles in $\mathbf{D}$ of total multiplicity $\kappa$ and that, for the function $F_{\beta}$ defined by extrapolation of (3.13) to $\widehat{\mathbf{D}}$ such that $F_{\beta}(z)=-\left(F_{\beta}\left(\bar{z}^{-1}\right)\right)^{*}$ holds, we have

$$
F_{\beta} \in C_{0, \kappa}^{+}(\mathscr{L}(\mathscr{H}))
$$

There exists an $\eta>0$ such that

$$
\lim _{\beta \rightarrow \infty}\left\|F_{\beta}(z)-F^{(\alpha)}(z)\right\|=0
$$

uniformly for $|z| \leq \eta$.

Let $\mu \in \mathbf{T}$. Then making use of (3.12), (3.14), (3.15) and Corollary 3.2 we see that for sufficiently small $\varepsilon>0$ there is a number $N(\varepsilon)$ such that for $\beta>N(\varepsilon)$

$$
r_{-}^{\mathbf{D}}\left(\delta_{\varepsilon} ; F_{\beta}\right)=r_{-}^{\mathbf{D}}\left(\delta_{\varepsilon} ; F^{(\alpha)}\right),
$$

where $\delta_{\varepsilon}:=\left\{z \in \overline{\mathbf{D}}: \operatorname{dist}_{\overline{\mathbf{C}}}(z, \mu)<\varepsilon\right\}$. Then the first part of the proof and the fact that the poles of $F_{\beta}$ in $\mathbf{D}$ coincide with the zeros of $F^{(\alpha+\beta)}$ in $\mathbf{D}$, according to their multiplicities (see (3.13)), yield the desired conclusion. 


\section{References}

[1] Aronszajn, N., and W.F. Donoghue, JR.: On exponential representations of analytic functions in the upper half-plane with positive imaginary part. - J. Analyse Math. 5, 1956/1957, 321-388.

[2] Berg, C., J.P.R. Christensen, and P.H. Maserick: Sequences with finitely many negative squares. - J. Funct. Anal. 79, 1988, 260-287.

[3] BRoDSKIĬ, M.S.: Triangular and Jordan representations of linear operators. - Translations of Mathematical Monographs 32. American Mathematical Society, Providence (N.J.), 1972.

[4] Daho, K., and H. Langer: Matrix functions of the class $N_{\kappa}$. - Math. Nachr. 120, 1985, 275-294.

[5] Dijksma, A., H. LANGer, and H.S.V. DE SNoo: Representations of holomorphic operator functions by means of resolvents of unitary or selfadjoint operators in Kreĭn spaces. - Operators in indefinite metric spaces, scattering theory and other topics (Bucharest, 1985). Operator Theory: Advances and Applications 24. Birkhäuser Verlag, Basel-Boston-Stuttgart, 1987, 123-143.

[6] Donoghue, W.F., JR.: Monotone matrix functions and analytic continuation. - Die Grundlehren der mathematischen Wissenschaften 207. Springer-Verlag, Berlin-Heidelberg-New York, 1974.

[7] Grothendieck, A.: Sur certains espaces de fonctions holomorphes. I. - J. Reine Angew. Math. 192, 1953, 35-64.

[8] IoHvidov, I.S., and M.G. KRĚ̃N: Spectral theory of operators in spaces with an indefinite metric. II. - Amer. Math. Soc. Transl. (2) 34, 1963, 283-373.

[9] Jonas, P.: Zur Existenz von Eigenspektralfunktionen mit Singularitäten. - Math. Nachr. 88, 1979, 345-361.

[10] Jonas, P.: On the functional calculus and the spectral function for definitizable operators in Krein space. - Beiträge Anal. 16, 1981, 121-135.

[11] Jonas, P.: On a class of unitary operators in Krein space. - Advances in invariant subspaces and other results of operator theory (Timişoara and Herculane, 1984). Operator Theory: Advances and Applications 17. Birkhäuser Verlag, Basel-BostonStuttgart, 1986, 151-172.

[12] Jonas, P.: On a class of selfadjoint operators in Krein space and their compact perturbations. - Integral Equations Operator Theory 11, 1988, 351-384.

[13] Jonas, P., H. Langer, and B. Textorius: Models and unitary equivalence of cyclic selfadjoint operators in Pontrjagin space. - To appear.

[14] Köтнe, G.: Die Randverteilungen analytischer Funktionen. - Math. Z. 57, 1952/1953, $13-33$.

[15] Köthe, G.: Topologische lineare Räume. I. - Die Grundlehren der mathematischen Wissenschaften 107. Springer-Verlag, Berlin-Göttingen-Heidelberg, 1960.

[16] Krě̌n, M.G., and H. LANGER: Über die verallgemeinerten Resolventen und die charakteristische Funktion eines isometrischen Operators im Raume $\Pi_{\kappa}$. - Hilbert space operators and operator algebras. Colloquia Mathematica Societatis János Bolyai 5. North-Holland Publishing Company, Amsterdam-London, 1972, 353-399.

[17] Krě̌s, M.G., and H. Langer: Über die $Q$-Funktion eines $\pi$-hermiteschen Operators im Raume $\Pi_{\kappa}$. - Acta Sci. Math. (Szeged) 34, 1973, 191-230. 
[18] Krěrs, M.G., and H. Langer: Über einige Fortsetzungsprobleme, die eng mit der Theorie hermitescher Operatoren im Raume $\Pi_{\kappa}$ zusammenhängen. I. Einige Funktionenklassen und ihre Darstellungen. - Math. Nachr. 77, 1977, 187-236.

[19] KreǏn, M.G., and H. LANGER: Some propositions on analytic matrix functions related to the theory of operators in the space $\Pi_{\kappa}$. - Acta Sci. Math. (Szeged) 43, 1981, 181-205.

[20] Langer, H.: Invariante Teilräume definisierbarer $J$-selbstadjungierter Operatoren. - Ann. Acad. Sci. Fenn. Ser. A I Math. 475, 1971, 1-23.

[21] Langer, H., and B. Najman: Perturbation theory of definitizable operators in Krein spaces. - J. Operator Theory 9, 1983, 297-317.

[22] ShtraUs, V.A. [B.A. Штраус]: О дефинизируемом аналоге проблемы моментов Хаусдорфа. - Akad. Nauk Armyan. SSR Dokl. 84:1, 1987, 9-12.

Received 12 August 1991 